\title{
Twisted vertex operators and unitary Lie algebras
}

\author{
Fulin Chen $^{3}$, Yun Gao ${ }^{1}$, Naihuan Jing ${ }^{2}$, Shaobin Tan ${ }^{3}$ \\ ${ }^{1}$ Department of Mathematics and Statistics, York University \\ Toronto, Canada M3J 1P3 \\ 2 Department of Mathematics, North Carolina State University \\ Raleigh, NC, USA 27695 \\ ${ }^{3}$ Department of Mathematics, Xiamen University \\ Xiamen, China 361005
}

\begin{abstract}
A representation of the central extension of the unitary Lie algebra coordinated with a skew Laurent polynomial ring is constructed using vertex operators over an integral $\mathbb{Z}_{2}$-lattice. The irreducible decomposition of the representation is explicitly computed and described. As a by-product, some fundamental representations of affine Kac-Moody Lie algebra of type $A_{n}^{(2)}$ are recovered by the new method.
\end{abstract}

\section{Introduction}

Affine Kac-Moody Lie algebras, or nontrivial central extensions of loop algebras, are a class of infinite dimensional Lie algebras fundamentally important in mathematics and theoretical physics. They are first realized by vertex operators in [LW] and [KKLW] for the principal picture and later in [FK, S] for the homogeneous picture. During the last two decades these constructions have been generalized from several directions. The vertex operator representations of the toroidal Lie algebras have been given in [MRY, Y, EM] and [T3] (see also [FJW] in the homogenous setting and in [B] and [T2 in the principle setting; vertex representations of quantum affine algebras have been obtained in [FJ] for untwisted cases and in [J] for twisted cases; the vertex representations of the extended affine Lie algebras of type $A$ coordinated by a quantum torus have been provided in [BS] and [G4] via the principle construction and in [G3] via the homogenous construction. Finally in [BGT] a unified treatment of vertex representations of affine Lie algebras using mixed bosons and fermions have been studied, and the Tits-Kantor-Koecher algebra has also been realized in [T1] using the vertex operator calculus.

On the other hand, before the development of toroidal Lie algebras, the elementary unitary Lie algebra $\mathfrak{e u}_{\nu}\left(\mathcal{R},{ }^{-}\right)$associated with an involutive associative algebra $\mathcal{R}$ was studied in $[\mathrm{AF}]$ as a derived subalgebra of the unitary Lie algebra $\mathfrak{u}_{\nu}\left(\mathcal{R},{ }^{-}\right)$. These Lie algebras are generalization of the usual loop algebras by replacing the commutative coordinate ring with an noncommutative algebra. As is well-known that the central extensions of Lie algebras are 
essentially given by A. Connes' cyclic homology $[\mathrm{KL}$. For the extended affine Lie algebras the central extensions are also described by the dihedral homology [G1, ABG], and the relevant central extensions in the unitary algebras coordinated with an involutive algebra will be given by a Steinberg Lie algebra structure.

The Steinberg group is the universal central extension of the commutator subgroup of the general linear group. The Steinberg Lie algebra is defined as an associative algebra generated by the generators $x_{i j}(\lambda)$ subject to the Steinberg relations. The extended affine Lie algebras share many common features with loop algebras and also have distinguished central extensions given by dihedral homology. When one further generalizes the algebraic structure by replacing the coordinate ring with the quantum polynomial ring or even an associative algebra, Steinberg unitary Lie algebras shall be the key for the universal central extensions. The realization of Steinberg unitary Lie algebras is thus a natural question in this regard.

In this paper, inspired by the work of Wakimoto in [W], we will construct a family of twisted vertex operators associated to an integral $\mathbb{Z}_{2}$-lattice of rank $\nu$ to realize the Steinberg relations. More precisely, for any non-zero complex number $a$, we define vertex operators $X_{i j}(a, z)$ for $1 \leq i, j \leq \nu$ on a Fock space $M$. For the purpose of computing the commutator relations, two multi-product decompositions of $\delta$-function and $D \delta$-function are developed. For any abelian group $G$ with a character $\sigma: G \rightarrow \mathbb{C}^{\times}$, we can define a corresponding skew Laurent-polynomial ring $\mathcal{R}_{\sigma}$ and an anti-involution ${ }^{-}$over $\mathcal{R}_{\sigma}$. We will show that the vertex operators $X_{i j}(a, z)$ give a representation for the Lie algebra $\widehat{\mathfrak{u}}_{\nu}\left(\mathcal{R}_{\sigma},{ }^{-}\right)$and also provide a nontrivial central extension of the unitary Lie algebra $\mathfrak{u}_{\nu}\left(\mathcal{R}_{\sigma}{ }^{-}\right)$associated to the pair $\left(\mathcal{R}_{\sigma}{ }^{-}\right)$, namely, the twisted vertex representation is actually a module for the central extension $\widehat{\mathfrak{e u}}_{\nu}\left(\mathcal{R}_{\sigma}{ }^{-}\right)$.

As was shown in [W], it is interesting to analyze the irreducible decomposition of the twisted module. Based on the observation that the twisted group algebra of the integral $\mathbb{Z}_{2}$-lattice is isomorphic to a (finite dimensional) Clifford algebra we are able to use the wellknown classical representation theory of finite dimensional Clifford algebra to achieve this goal. We remark that this is slightly different from the approach taken by Wakimoto in W]. Let $|\sigma|$ be the order of the group $\sigma(G)$, if $|\sigma|=\infty$ or $|\sigma| \in 2 \mathbb{N}$, then each irreducible component of $\widehat{\mathfrak{u}}_{\nu}\left(\mathcal{R}_{\sigma},{ }^{-}\right)$-module $M$ remains irreducible as $\widehat{\mathfrak{e u}}_{\nu}\left(\mathcal{R}_{\sigma},{ }^{-}\right)$-module. For the case $|\sigma| \in 2 \mathbb{N}+1$, note that in this case all the elements in $\sigma(G)$ are roots of unity. To this end, we introduce a conjugate anti-involution $\tau$ on $\widehat{\mathfrak{u}}_{\nu}\left(\mathcal{R}_{\sigma},{ }^{-}\right)$as all the elements of $\sigma(G)$ lie in the unit circle in the complex plane. Then, we prove that the $\widehat{\mathfrak{u}}_{\nu}\left(\mathcal{R}_{\sigma}{ }^{-}\right)$-module $M$ is unitary with respect to $\tau$, which in turn deduce that $M$ is completely reducible as $\widehat{\mathfrak{e u}}_{\nu}\left(\mathcal{R}_{\sigma}{ }^{-}{ }^{-}\right.$)-module even if $|\sigma| \in 2 \mathbb{N}+1$. As a by-product when taking $G=\{1\}$, we get a completely reducible module $M$ for the affine Kac-Moody Lie algebra of type $A_{\nu-1}^{(2)}$, which recovers the result in W].

The paper is organized as follows. In Sect.2, we define a family of vertex operators for any $a \in \mathbb{C}^{\times}$and then compute their commutation relations. In Sect.3, using the derived relations we construct the vertex operator representations for the unitary Lie algebra with 
non-trivial central extension and then determine the irreducible components. Finally, in Sect.4, we construct vertex representations for the elementary unitary Lie algebra with nontrivial central extension. As an example we also obtain a new realization of the affine KacMoody algebra of type $A_{\nu-1}^{(2)}$.

Throughout this paper, we denote the field of complex numbers, the group of non-zero complex numbers, the ring of integers and the set of non-negative integers by $\mathbb{C}, \mathbb{C}^{\times}, \mathbb{Z}$ and $\mathbb{N}$, respectively.

\section{Fock Space and Vertex Operators}

Let $\nu \geq 2$ be a positive integer. Let $\Gamma=\oplus_{i=1}^{\nu} \mathbb{Z} \epsilon_{i},\left(\epsilon_{i}, \epsilon_{j}\right)=\delta_{i j}$ for $i, j=1, \cdots, \nu$, and $\epsilon_{i}(n)$ be a linear copy of $\epsilon_{i}$ for $n \in 2 \mathbb{Z}+1$ and $i=1, \cdots, \nu$. We define a Lie algebra

$$
\mathcal{H}=\operatorname{span}_{\mathbb{C}}\left\{\epsilon_{i}(n), 1 \mid n \in 2 \mathbb{Z}+1\right\}
$$

subject to the following Lie algebra relation

$$
[\alpha(m), \beta(n)]=\frac{m}{2}(\alpha, \beta) \delta_{m+n, 0}
$$

for $\alpha, \beta \in \Gamma$, and $m, n \in 2 \mathbb{Z}+1$. Let $\bar{\Gamma}=\Gamma / 2 \Gamma$ be the quotient additive group of $\Gamma$ factored by the subgroup $2 \Gamma$. Let $\mathbb{C}[\bar{\Gamma}]=\oplus_{\alpha \in \bar{\Gamma}} \mathbb{C} e^{\alpha}$ be the twisted group over the finite group $\bar{\Gamma}$

with multiplication defined by $e^{\bar{\alpha}} e^{\bar{\beta}}=\varepsilon(\alpha, \beta) e^{\overline{\alpha+\beta}}$ for $\alpha, \beta \in \Gamma$, where the two-cocycle $\varepsilon$ : $\Gamma \times \Gamma \rightarrow\{ \pm 1\}$ is given by

$$
\varepsilon\left(\epsilon_{i}, \epsilon_{j}\right)= \begin{cases}1, & \text { if } i \leq j \\ -1, & \text { if } i>j\end{cases}
$$

and

$$
\varepsilon\left(\sum m_{i} \epsilon_{i}, \sum n_{j} \epsilon_{j}\right)=\prod_{i, j}\left(\varepsilon\left(\epsilon_{i}, \epsilon_{j}\right)\right)^{m_{i} n_{j}} .
$$

We note that the multiplication in $\mathbb{C}[\bar{\Gamma}]$ is well-defined as $\varepsilon\left(\alpha_{1}, \beta_{1}\right)=\varepsilon\left(\alpha_{2}, \beta_{2}\right)$ if $\alpha_{2}-\alpha_{1}, \beta_{2}-$ $\beta_{1} \in 2 \Gamma$. Let $z, w$ be formal variables and $\alpha \in \Gamma$, set

$$
E_{ \pm}(\alpha, z)=\exp \left(-2 \sum_{n \in \pm(2 \mathbb{N}+1)} \frac{\alpha(n)}{n} z^{-n}\right)
$$

and define the Fock space

$$
M=\mathbb{C}[\bar{\Gamma}] \otimes \mathcal{S}\left(\mathcal{H}^{-}\right)
$$

where $\mathcal{S}\left(\mathcal{H}^{-}\right)$is the usual commutative symmetric algebra over the subalgebra $\mathcal{H}^{-}$of $\mathcal{H}$ spanned by $\epsilon_{i}(n)$ with $1 \leq i \leq \nu$ and $n \in-(2 \mathbb{N}+1)$.

Lemma 2.1 For $\alpha, \beta \in \Gamma$, one has

$$
E_{ \pm}(\alpha, z)=E_{ \pm}(-\alpha,-z)
$$




$$
E_{+}(\alpha, z) E_{-}(\beta, w)=E_{-}(\beta, w) E_{+}(\alpha, z)\left(\frac{1-w / z}{1+w / z}\right)^{(\alpha, \beta)}
$$

Proof. The first identity is clear. To prove the second identity, we set

$$
A=-2 \sum_{n \in(2 \mathbb{N}+1)} \frac{\alpha(n)}{n} z^{-n}, \quad B=-2 \sum_{n \in-(2 \mathbb{N}+1)} \frac{\beta(n)}{n} w^{-n},
$$

and note that

$$
\begin{gathered}
{[A, B]=4 \sum_{m \in(2 \mathbb{N}+1)} \sum_{n \in-(2 \mathbb{N}+1)} \frac{z^{-m} w^{-n}}{m n}[\alpha(m), \beta(n)]} \\
=4 \sum_{m \in(2 \mathbb{N}+1)} \frac{z^{-m} w^{m}}{-m^{2}}(\alpha, \beta) \frac{m}{2} \\
=-2(\alpha, \beta) \sum_{m \in(2 \mathbb{N}+1)} \frac{1}{m}(w / z)^{m}=\log \left(\frac{1-w / z}{1+w / z}\right)^{(\alpha, \beta)} .
\end{gathered}
$$

Then by applying the formal rule $e^{A} e^{B}=e^{[A, B]} e^{B} e^{A}$ if $[A, B]$ commutes with $A$ and $B$, we get the required identity.

For $a \in \mathbb{C}^{\times}$, we define the following vertex operators, which act on the Fock space $M$ as the usual way (see $[\mathrm{FLM}]$ ).

$$
\begin{gathered}
X_{i j}(a, z)= \\
\begin{cases}\varepsilon\left(\epsilon_{i}, \epsilon_{j}\right) e^{\overline{\epsilon_{i}-\epsilon_{j}}} E_{-}\left(\epsilon_{i}, z\right) E_{-}\left(-\epsilon_{j}, a z\right) E_{+}\left(\epsilon_{i}, z\right) E_{+}\left(-\epsilon_{j}, a z\right), & i \neq j ; \\
4 \epsilon_{i}(z), & i=j, a=1 ; \\
\frac{1+a}{1-a}\left(E_{-}\left(\epsilon_{i}, z\right) E_{-}\left(-\epsilon_{i}, a z\right) E_{+}\left(\epsilon_{i}, z\right) E_{+}\left(-\epsilon_{i}, a z\right)-1\right), & i=j, a \neq 1 .\end{cases}
\end{gathered}
$$

where $\epsilon_{i}(z)=\sum_{n \in 2 \mathbb{Z}+1} \epsilon_{i}(n) z^{-n}$, and $e^{\overline{\epsilon_{i}-\epsilon_{j}}}$ is the usual operator acting on the group algebra $\mathbb{C}[\bar{\Gamma}]$ twisted by the two-cocycle $\varepsilon$. Set

$$
A_{ \pm}=-2 \sum_{n \in \pm(2 \mathbb{N}+1)} \frac{\epsilon_{i}(n)}{n}(a z)^{-n}, \quad B_{ \pm}=-2 \sum_{n \in \pm(2 \mathbb{N}+1)} \frac{\epsilon_{i}(n)}{n} z^{-n}
$$


Then for $a \neq 1$, we have

$$
\begin{aligned}
& \frac{1}{1-a}\left(E_{ \pm}\left(\epsilon_{i}, z\right)-E_{ \pm}\left(\epsilon_{i}, a z\right)\right) \\
= & \frac{1}{1-a}\left(e^{B_{ \pm}}-e^{A_{ \pm}}\right)=\frac{1}{1-a}\left(\sum_{l=1}^{\infty} \frac{1}{l !} B_{ \pm}^{l}-\sum_{l=1}^{\infty} \frac{1}{l !} A_{ \pm}^{l}\right) \\
= & \frac{1}{1-a} \sum_{l=1}^{\infty} \frac{1}{l !}\left[\left(2 \sum_{n \in \pm(2 \mathbb{N}+1)} \frac{\epsilon_{i}(n)}{n}(a z)^{-n}-2 \sum_{n \in \pm(2 \mathbb{N}+1)} \frac{\epsilon_{i}(n)}{n} z^{-n}\right) \sum_{j=0}^{l-1} A_{ \pm}^{l-1-j} B_{ \pm}^{j}\right] \\
= & \sum_{l=1}^{\infty} \frac{1}{l !}\left[2 \sum_{n \in \pm(2 \mathbb{N}+1)} \frac{\epsilon_{i}(n)}{n}(z)^{-n} \frac{a^{-n}-1}{1-a} \sum_{j=0}^{l-1} A_{ \pm}^{l-1-j} B_{ \pm}^{j}\right] \\
\longrightarrow & 2 \sum_{n \in \pm(2 \mathbb{N}+1)} \epsilon_{i}(n) z^{-n} \sum_{l=1}^{\infty} \frac{1}{l !} l B_{ \pm}^{l-1}=2 \sum_{n \in \pm(2 \mathbb{N}+1)} \epsilon_{i}(n) z^{-n} E_{ \pm}\left(\epsilon_{i}, z\right),
\end{aligned}
$$

as $a \rightarrow 1$. From this we obtain, for $a \neq 1$, that

$$
\begin{aligned}
X_{i i}(a, z)= & \frac{1+a}{1-a} E_{-}\left(-\epsilon_{i}, a z\right)\left(E_{-}\left(\epsilon_{i}, z\right) E_{+}\left(\epsilon_{i}, z\right)-E_{-}\left(\epsilon_{i}, a z\right) E_{+}\left(\epsilon_{i}, a z\right)\right) E_{+}\left(-\epsilon_{i}, a z\right) \\
= & (1+a) E_{-}\left(-\epsilon_{i}, a z\right)\left(\frac{E_{-}\left(\epsilon_{i}, z\right)-E_{-}\left(\epsilon_{i}, a z\right)}{1-a} E_{+}\left(\epsilon_{i}, z\right)+\right. \\
& \left.E_{-}\left(\epsilon_{i}, a z\right) \frac{E_{+}\left(\epsilon_{i}, z\right)-E_{+}\left(\epsilon_{i}, a z\right)}{1-a}\right) E_{+}\left(-\epsilon_{i}, a z\right) \\
\longrightarrow & 4 \epsilon_{i}(z)
\end{aligned}
$$

as $a \rightarrow 1$. Therefore, we have

$$
\lim _{a \rightarrow 1} X_{i i}(a, z)=X_{i i}(1, z) .
$$

Moreover, from the definition of the vertex operator $X_{i j}(a, z)$, we can easily check the following result

Lemma 2.2 For $a \in \mathbb{C}^{\times}$, and $1 \leq i, j \leq \nu$, one has

$$
X_{i j}(a, z)=-X_{j i}\left(a^{-1},-a z\right) .
$$

The vertex operator $X_{i j}(a, z)$ can be formally expanded as follows

$$
X_{i j}(a, z)=\sum_{n \in \mathbb{Z}} x_{i j}(a, n) z^{-n}
$$

where $x_{i j}(a, n)$ are operators acting on the Fock space $M$. Then the equation in the previous lemma implies that

$$
x_{j i}\left(a^{-1}, n\right)=-(-1)^{n} a^{n} x_{i j}(a, n),
$$

for $n \in \mathbb{Z}, i, j=1, \cdots, \nu, a \in \mathbb{C}^{\times}$. 
Lemma 2.3 For $\alpha \in \Gamma$, set $\alpha(z)=\sum_{n \in 2 \mathbb{Z}+1} \alpha(n) z^{-n}$, then

$$
\left[\alpha(z), E_{ \pm}(\beta, w)\right]=(\alpha, \beta) E_{ \pm}(\beta, w) \sum_{n \in \pm(2 \mathbb{N}+1)}\left(\frac{z}{w}\right)^{n}
$$

Proof. The result follows from the formal rule $\left[A, e^{B}\right]=[A, B] e^{B}$ if $[A, B]$ commutes with $B$, and the identity

$$
\left[\alpha(z),-2 \sum_{n \in \pm(2 \mathbb{N}+1)} \frac{\beta(n)}{n} w^{-n}\right]=(\alpha, \beta) \sum_{n \in \pm(2 \mathbb{N}+1)}\left(\frac{z}{w}\right)^{n} .
$$

The following result is well-known (see $[\mathrm{FLM}]$ ), and will be used frequently later on.

Lemma 2.4 Let $Y(w, z)$ be a formal power series in $w, z$ with coefficient in a vector space, such that $\lim _{z \rightarrow w} Y(w, z)$ exists (in the sense of [FLM]). Set $D_{z}=z \frac{\partial}{\partial z}$, then

$$
\begin{aligned}
Y(w, z) \delta\left(a \frac{w}{z}\right) & =Y(w, a w) \delta\left(a \frac{w}{z}\right) \\
Y(w, z)(D \delta)\left(a \frac{w}{z}\right) & =Y(w, a w)(D \delta)\left(a \frac{w}{z}\right)+\left(D_{z} Y\right)(w, z) \delta\left(a \frac{w}{z}\right) .
\end{aligned}
$$

Now we are going to compute the Lie product $\left[X_{i j}(a, z), X_{k l}(b, w)\right]$ for $1 \leq i, j, k, l \leq \nu$, and $a, b \in \mathbb{C}^{\times}$. For this purpose we need the following several combinatorial identities

Lemma 2.5 Let $B$ and $A_{i}$ for $i=0,1, \cdots, n$ be non-zero distinct complex numbers, then

$$
\begin{aligned}
& \left(\frac{B}{B-A_{0}}\right)^{2} \prod_{j=1}^{n} \frac{B}{B-A_{j}}=\sum_{i=1}^{n}\left(\frac{A_{i}}{A_{i}-A_{0}}\right)^{2}\left(\prod_{1 \leq j \leq n, j \neq i} \frac{A_{i}}{A_{i}-A_{j}}\right) \frac{B}{B-A_{i}} \\
& +\left(\prod_{i=1}^{n} \frac{A_{0}}{A_{0}-A_{i}}\right)\left[\left(\frac{B}{B-A_{0}}\right)^{2}+\left(\sum_{l=1}^{n} \frac{A_{l}}{A_{l}-A_{0}}\right) \frac{B}{B-A_{0}}\right], \\
& \left(\frac{A_{0}}{B-A_{0}}\right)^{2} \prod_{j=1}^{n} \frac{A_{j}}{B-A_{j}}=\sum_{i=1}^{n}\left(\frac{A_{0}}{A_{i}-A_{0}}\right)^{2}\left(\prod_{1 \leq j \leq n, j \neq i} \frac{A_{j}}{A_{i}-A_{j}}\right) \frac{A_{i}}{B-A_{i}} \\
& +\left(\prod_{i=1}^{n} \frac{A_{i}}{A_{0}-A_{i}}\right)\left[\left(\frac{A_{0}}{B-A_{0}}\right)^{2}+\left(\sum_{l=1}^{n} \frac{A_{0}}{A_{l}-A_{0}}\right) \frac{A_{0}}{B-A_{0}}\right] .
\end{aligned}
$$

for $n \geq 1$.

Proof. We will only prove the first identity by induction on $n$. The proof of the second one is similar to that of the first one, and we omit it. 
It is not difficult to check the result for $n=1$. For the case $n+1$, we have

$$
\begin{aligned}
& \sum_{i=1}^{n+1}\left(\frac{A_{i}}{A_{i}-A_{0}}\right)^{2}\left(\prod_{1 \leq j \leq n+1, j \neq i} \frac{A_{i}}{A_{i}-A_{j}}\right) \frac{B}{B-A_{i}}+ \\
& \left(\prod_{i=1}^{n+1} \frac{A_{0}}{A_{0}-A_{i}}\right)\left[\left(\frac{B}{B-A_{0}}\right)^{2}+\left(\sum_{l=1}^{n+1} \frac{A_{l}}{A_{l}-A_{0}}\right) \frac{B}{B-A_{0}}\right] \\
& =\left(\frac{A_{n+1}}{A_{n+1}-A_{0}}\right)^{2}\left(\prod_{j=1}^{n} \frac{A_{n+1}}{A_{n+1}-A_{j}}\right) \frac{B}{B-A_{n+1}} \\
& +\sum_{i=1}^{n}\left(\frac{A_{i}}{A_{i}-A_{0}}\right)^{2}\left(\prod_{1 \leq j \leq n, j \neq i} \frac{A_{i}}{A_{i}-A_{j}}\right) \frac{A_{i}}{A_{i}-A_{n+1}} \frac{B}{B-A_{i}} \\
& +\left(\prod_{i=1}^{n} \frac{A_{0}}{A_{0}-A_{i}}\right)\left[\left(\frac{A_{0}}{A_{0}-A_{n+1}} \frac{B}{B-A_{0}}\right)\left(\frac{B}{B-A_{0}}-\frac{A_{n+1}}{A_{0}-A_{n+1}}+\sum_{l=1}^{n} \frac{A_{l}}{A_{l}-A_{0}}\right)\right] \\
& =\left(\frac{A_{n+1}}{A_{n+1}-A_{0}}\right)^{2}\left(\prod_{j=1}^{n} \frac{A_{n+1}}{A_{n+1}-A_{j}}\right) \frac{B}{B-A_{n+1}} \\
& +\sum_{i=1}^{n}\left(\frac{A_{i}}{A_{i}-A_{0}}\right)^{2}\left(\prod_{1 \leq j \leq n, j \neq i} \frac{A_{i}}{A_{i}-A_{j}}\right)\left(\frac{A_{n+1}}{A_{i}-A_{n+1}}+\frac{B}{B-A_{i}}\right) \frac{B}{B-A_{n+1}} \\
& +\left(\prod_{i=1}^{n} \frac{A_{0}}{A_{0}-A_{i}}\right)\left[\left(\frac{B}{B-A_{0}}\right)^{2}-\left(\frac{A_{n+1}}{A_{0}-A_{n+1}}\right)^{2}\right] \frac{B}{B-A_{n+1}} \\
& +\left(\prod_{i=1}^{n} \frac{A_{0}}{A_{0}-A_{i}}\right)\left(\sum_{l=1}^{n} \frac{A_{l}}{A_{l}-A_{0}}\right)\left(\frac{A_{n+1}}{A_{0}-A_{n+1}}+\frac{B}{B-A_{0}}\right) \frac{B}{B-A_{n+1}} \\
& =\left(\frac{A_{n+1}}{A_{n+1}-A_{0}}\right)^{2}\left(\prod_{j=1}^{n} \frac{A_{n+1}}{A_{n+1}-A_{j}}\right) \frac{B}{B-A_{n+1}}+F_{1}+F_{2},
\end{aligned}
$$

where

$$
\begin{aligned}
F_{1}= & \sum_{i=1}^{n}\left(\frac{A_{i}}{A_{i}-A_{0}}\right)^{2}\left(\prod_{1 \leq j \leq n, j \neq i} \frac{A_{i}}{A_{i}-A_{j}}\right)\left(\frac{A_{n+1}}{A_{i}-A_{n+1}}\right) \frac{B}{B-A_{n+1}} \\
& +\left(\prod_{i=1}^{n} \frac{A_{0}}{A_{0}-A_{i}}\right)\left[\left(\sum_{l=1}^{n} \frac{A_{l}}{A_{l}-A_{0}}\right) \frac{A_{n+1}}{A_{0}-A_{n+1}}-\left(\frac{A_{n+1}}{A_{0}-A_{n+1}}\right)^{2}\right] \frac{B}{B-A_{n+1}}, \\
F_{2}= & \sum_{i=1}^{n}\left(\frac{A_{i}}{A_{i}-A_{0}}\right)^{2}\left(\prod_{1 \leq j \leq n, j \neq i} \frac{A_{i}}{A_{i}-A_{j}}\right)\left(\frac{B}{B-A_{i}}\right) \frac{B}{B-A_{n+1}} \\
& +\left(\prod_{i=1}^{n} \frac{A_{0}}{A_{0}-A_{i}}\right)\left[\left(\frac{B}{B-A_{0}}\right)^{2}+\left(\sum_{l=1}^{n} \frac{A_{l}}{A_{l}-A_{0}}\right) \frac{B}{B-A_{0}}\right] \frac{B}{B-A_{n+1}}
\end{aligned}
$$


Now, by using induction on $n$, we find

$$
\begin{aligned}
& F_{1}=-\left(\frac{A_{n+1}}{A_{n+1}-A_{0}}\right)^{2}\left(\prod_{j=1}^{n} \frac{A_{n+1}}{A_{n+1}-A_{j}}\right) \frac{B}{B-A_{n+1}}, \\
& F_{2}=\left(\frac{B}{B-A_{0}}\right)^{2} \prod_{j=1}^{n+1} \frac{B}{B-A_{j}},
\end{aligned}
$$

as required.

Corollary 2.6 Let $A_{i} \neq A_{j}$ for $i \neq j$ be nonzero complex numbers, then

$$
\begin{aligned}
& \left(\frac{1}{1-A_{0} x}\right)^{2} \prod_{j=1}^{n} \frac{1}{1-A_{j} x}=\sum_{i=1}^{n}\left(\frac{A_{i}}{A_{i}-A_{0}}\right)^{2}\left(\prod_{1 \leq j \leq n, j \neq i} \frac{A_{i}}{A_{i}-A_{j}}\right) \frac{1}{1-A_{i} x} \\
& +\left(\prod_{i=1}^{n} \frac{A_{0}}{A_{0}-A_{i}}\right)\left[\frac{A_{0} x}{\left(1-A_{0} x\right)^{2}}+\left(1+\sum_{l=1}^{n} \frac{A_{l}}{A_{l}-A_{0}}\right) \frac{1}{1-A_{0} x}\right], \\
& \left(\frac{A_{0} x}{1-A_{0} x}\right)^{2} \prod_{j=1}^{n} \frac{A_{j} x}{1-A_{j} x}=\sum_{i=1}^{n}\left(\frac{A_{0}}{A_{i}-A_{0}}\right)^{2}\left(\prod_{1 \leq j \leq n, j \neq i} \frac{A_{j}}{A_{i}-A_{j}}\right) \frac{A_{i} x}{1-A_{i} x} \\
& +\left(\prod_{i=1}^{n} \frac{A_{i}}{A_{0}-A_{i}}\right)\left[\frac{A_{0} x}{\left(1-A_{0} x\right)^{2}}+\left(\sum_{l=1}^{n} \frac{A_{0}}{A_{l}-A_{0}}-1\right) \frac{A_{0} x}{1-A_{0} x}\right] .
\end{aligned}
$$

Proposition 2.7 Let $A_{i}$ for $0 \leq i \leq n$ be distinct nonzero complex numbers, then

$$
\begin{aligned}
& \left(\frac{1}{1-A_{0} x}\right)^{2} \prod_{i=1}^{n} \frac{1}{1-A_{i} x}-(-1)^{n}\left(\frac{A_{0}^{-1} x^{-1}}{1-A_{0}^{-1} x^{-1}}\right)^{2} \prod_{i=1}^{n} \frac{A_{i}^{-1} x^{-1}}{1-A_{i}^{-1} x^{-1}} \\
= & \sum_{i=1}^{n}\left[\left(\frac{A_{i}}{A_{i}-A_{0}}\right)^{2} \prod_{1 \leq j \leq n, j \neq i} \frac{A_{i}}{A_{i}-A_{j}}\right] \delta\left(A_{i} x\right) \\
& +\left(\prod_{i=1}^{n} \frac{A_{0}}{A_{0}-A_{i}}\right)\left[(D \delta)\left(A_{0} x\right)+\left(1+\sum_{l=1}^{n} \frac{A_{l}}{A_{l}-A_{0}}\right) \delta\left(A_{0} x\right)\right]
\end{aligned}
$$


Proof. By applying the second identity in Corollary 2.6, we have

$$
\begin{aligned}
& (-1)^{n-1}\left(\frac{A_{0}^{-1} x^{-1}}{1-A_{0}^{-1} x^{-1}}\right)^{2} \prod_{i=1}^{n} \frac{A_{i}^{-1} x^{-1}}{1-A_{i}^{-1} x^{-1}} \\
= & (-1)^{n-1} \sum_{i=1}^{n}\left(\frac{A_{0}^{-1}}{A_{i}^{-1}-A_{0}^{-1}}\right)^{2}\left(\prod_{1 \leq j \leq n, j \neq i} \frac{A_{j}^{-1}}{A_{i}^{-1}-A_{j}^{-1}}\right) \frac{A_{i}^{-1} x^{-1}}{1-A_{i}^{-1} x^{-1}} \\
& +(-1)^{n-1}\left(\prod_{i=1}^{n} \frac{A_{i}^{-1}}{A_{0}^{-1}-A_{i}^{-1}}\right)\left[\frac{A_{0}^{-1} x^{-1}}{\left(1-A_{0}^{-1} x^{-1}\right)^{2}}+\left(\sum_{l=1}^{n} \frac{A_{0}^{-1}}{A_{l}^{-1}-A_{0}^{-1}}-1\right) \frac{A_{0}^{-1} x^{-1}}{1-A_{0}^{-1} x^{-1}}\right] \\
= & \sum_{i=1}^{n}\left(\frac{A_{i}}{A_{i}-A_{0}}\right)^{2}\left(\prod_{1 \leq j \leq n, j \neq i} \frac{A_{i}}{A_{i}-A_{j}}\right) \frac{A_{i}^{-1} x^{-1}}{1-A_{i}^{-1} x^{-1}} \\
& -\left(\prod_{i=1}^{n} \frac{A_{0}}{A_{0}-A_{i}}\right)\left[\frac{A_{0}^{-1} x^{-1}}{\left(1-A_{0}^{-1} x^{-1}\right)^{2}}-\left(1+\sum_{l=1}^{n} \frac{A_{l}}{A_{l}-A_{0}}\right) \frac{A_{0}^{-1} x^{-1}}{1-A_{0}^{-1} x^{-1}}\right]
\end{aligned}
$$

Therefore, from the first identity in Corollary 2.6, we obtain

$$
\begin{aligned}
& \left(\frac{1}{1-A_{0} x}\right)^{2} \prod_{i=1}^{n} \frac{1}{1-A_{i} x}-(-1)^{n}\left(\frac{A_{0}^{-1} x^{-1}}{1-A_{0}^{-1} x^{-1}}\right)^{2} \prod_{i=1}^{n} \frac{A_{i}^{-1} x^{-1}}{1-A_{i}^{-1} x^{-1}} \\
= & \sum_{i=1}^{n}\left(\frac{A_{i}}{A_{i}-A_{0}}\right)^{2}\left(\prod_{1 \leq j \leq n, j \neq i} \frac{A_{i}}{A_{i}-A_{j}}\right)\left(\frac{1}{1-A_{i} x}+\frac{A_{i}^{-1} x^{-1}}{1-A_{i}^{-1} x^{-1}}\right) \\
& +\left(\prod_{i=1}^{n} \frac{A_{0}}{A_{0}-A_{i}}\right)\left(\frac{A_{0} x}{\left(1-A_{0} x\right)^{2}}-\frac{A_{0}^{-1} x^{-1}}{\left(1-A_{0}^{-1} x^{-1}\right)^{2}}\right) \\
& +\left(\prod_{i=1}^{n} \frac{A_{0}}{A_{0}-A_{i}}\right)\left[\left(1+\sum_{l=1}^{n} \frac{A_{l}}{A_{l}-A_{0}}\right)\left(\frac{1}{1-A_{0} x}+\frac{A_{0}^{-1} x^{-1}}{1-A_{0} i^{-1} x^{-1}}\right)\right] \\
= & \sum_{i=1}^{n}\left[\left(\frac{A_{i}}{A_{i}-A_{0}}\right)^{2} \prod_{1 \leq j \leq n, j \neq i} \frac{A_{i}}{A_{i}-A_{j}}\right] \delta\left(A_{i} x\right) \\
& +\left(\prod_{i=1}^{n} \frac{A_{0}}{A_{0}-A_{i}}\right)\left[(D \delta)\left(A_{0} x\right)+\left(1+\sum_{l=1}^{n} \frac{A_{l}}{A_{l}-A_{0}}\right) \delta\left(A_{0} x\right)\right]
\end{aligned}
$$

Proposition 2.8 Let $A_{i}$ for $1 \leq i \leq n$ be distinct nonzero complex numbers, then

$$
\prod_{i=1}^{n} \frac{1}{1-A_{i} x}-(-1)^{n} \prod_{i=1}^{n} \frac{A_{i}^{-1} x^{-1}}{1-A_{i}^{-1} x^{-1}}=\sum_{i=1}^{n}\left(\prod_{1 \leq j \leq n, j \neq i} \frac{A_{i}}{A_{i}-A_{j}}\right) \delta\left(A_{i} x\right)
$$

Proof. By using Proposition 2.7 and the fact that

$$
\left(1-A_{0} x\right)^{2}\left(\frac{1}{1-A_{0} x}\right)^{2}=1=\left(1-A_{0} x\right)^{2}\left(\frac{A_{0}^{-1} x^{-1}}{1-A_{0}^{-1} x^{-1}}\right)^{2},
$$


one may get that,

$$
\begin{aligned}
& \prod_{i=1}^{n} \frac{1}{1-A_{i} x}-(-1)^{n} \prod_{i=1}^{n} \frac{A_{i}^{-1} x^{-1}}{1-A_{i}^{-1} x^{-1}} \\
= & \left(1-A_{0} x\right)^{2} \sum_{i=1}^{n}\left[\left(\frac{A_{i}}{A_{i}-A_{0}}\right)^{2} \prod_{1 \leq j \leq n, j \neq i} \frac{A_{i}}{A_{i}-A_{j}}\right] \delta\left(A_{i} x\right) \\
& +\left(1-A_{0} x\right)^{2}\left(\prod_{i=1}^{n} \frac{A_{0}}{A_{0}-A_{i}}\right)\left[(D \delta)\left(A_{0} x\right)+\left(1+\sum_{l=1}^{n} \frac{A_{l}}{A_{l}-A_{0}}\right) \delta\left(A_{0} x\right)\right] \\
= & \sum_{i=1}^{n}\left(\prod_{1 \leq j \leq n, j \neq i} \frac{A_{i}}{A_{i}-A_{j}}\right) \delta\left(A_{i} x\right)
\end{aligned}
$$

where have used Lemma 2.4 for the second equation.

For convenience, we set

$$
\begin{gathered}
: X_{i j}(a, z) X_{k l}(b, w):=e^{\overline{\epsilon_{i}-\epsilon_{j}+\epsilon_{k}-\epsilon_{l}}} E_{-}\left(\epsilon_{i}, z\right) E_{-}\left(-\epsilon_{j}, a z\right) E_{-}\left(\epsilon_{k}, w\right) E_{-}\left(-\epsilon_{l}, b w\right) \\
\cdot E_{+}\left(\epsilon_{i}, z\right) E_{+}\left(-\epsilon_{j}, a z\right) E_{+}\left(\epsilon_{k}, w\right) E_{+}\left(-\epsilon_{l}, b w\right)
\end{gathered}
$$

where $1 \leq i, j, k, l \leq \nu, a, b \in \mathbb{C}^{\times}$with the condition that $i \neq j$ if $a=1$ and $k \neq l$ if $b=1$. The proof of the following results is straightforward, and is omitted

Lemma 2.9 For $a, b \in \mathbb{C}^{\times}$and $\alpha \in \Gamma$, one has

$$
\begin{aligned}
{\left[X_{i j}(a, z), e^{\bar{\alpha}}\right] } & =\left((-1)^{\left(\epsilon_{i}-\epsilon_{j}, \alpha\right)}-1\right) e^{\bar{\alpha}} X_{i j}(a, z), \\
X_{i j}(a, z) X_{k l}(b, w) & =: X_{i j}(a, z) X_{k l}(b, w): P_{k l}^{i j}
\end{aligned}
$$

where $i \neq j$ if $a=1$ and $k \neq l$ if $b=1$, and

$$
\begin{gathered}
P_{k l}^{i j}=\varepsilon\left(\epsilon_{i}, \epsilon_{j}\right) \varepsilon\left(\epsilon_{k}, \epsilon_{l}\right) \varepsilon\left(\epsilon_{i}-\epsilon_{j}, \epsilon_{k}-\epsilon_{l}\right)\left(\frac{1-w / a z}{1+w / a z}\right)^{-\delta_{j k}} \\
\cdot\left(\frac{1-w / z}{1+w / z}\right)^{\delta_{i k}}\left(\frac{1-b w / a z}{1+b w / a z}\right)^{\delta_{j l}}\left(\frac{1-b w / z}{1+b w / z}\right)^{-\delta_{i l}} .
\end{gathered}
$$

By symmetry, from the previous lemma and (2.1) we obtain

Lemma 2.10 Let $i \neq j$ if $a=1$ and $k \neq l$ if $b=1$, then

$$
\left[X_{i j}(a, z), X_{k l}(b, w)\right]=: X_{i j}(a, z) X_{k l}(b, w): \Delta_{k l}^{i j},
$$


where

$$
\begin{aligned}
\Delta_{k l}^{i j}= & \left(\frac{1+a}{1-a}\right)^{\delta_{i j}}\left(\frac{1+b}{1-b}\right)^{\delta_{k l}} \varepsilon\left(\epsilon_{i}, \epsilon_{j}\right) \varepsilon\left(\epsilon_{k}, \epsilon_{l}\right) \varepsilon\left(\epsilon_{i}-\epsilon_{j}, \epsilon_{k}-\epsilon_{l}\right) \\
& \cdot\left(\frac{a z+w}{a z}\right)^{\delta_{j k}}\left(\frac{z-w}{z}\right)^{\delta_{i k}}\left(\frac{a z-b w}{a z}\right)^{\delta_{j l}}\left(\frac{z+b w}{z}\right)^{\delta_{i l}} Q_{k l}^{i j},
\end{aligned}
$$

and

$$
\begin{aligned}
Q_{k l}^{i j} & =\left(\frac{1}{1+\frac{w}{z}}\right)^{\delta_{i k}}\left(\frac{1}{1-\frac{w}{a z}}\right)^{\delta_{j k}}\left(\frac{1}{1-\frac{b w}{z}}\right)^{\delta_{i l}}\left(\frac{1}{1+\frac{b w}{a z}}\right)^{\delta_{j l}} \\
& -\left(\frac{\frac{z}{w}}{1+\frac{z}{w}}\right)^{\delta_{i k}}\left(\frac{-\frac{a z}{w}}{1-\frac{a z}{w}}\right)^{\delta_{j k}}\left(\frac{-\frac{z}{b w}}{1-\frac{z}{b w}}\right)^{\delta_{i l}}\left(\frac{\frac{a z}{b w}}{1+\frac{a z}{b w}}\right)^{\delta_{j l}} .
\end{aligned}
$$

In the rest of this section, we are going to compute the Lie product $\left[X_{i j}(a, z), X_{k l}(b, w)\right]$ with $1 \leq i, j, k, l \leq \nu$, and $a, b \in \mathbb{C}^{\times}$, where the vertex operators are defined by (2.3). For this purpose, we divide the argument into the following three cases:

Case one: $i \neq j, k \neq l$;

Case two: $i=j, k \neq l$;

Case three: $i=j, k=l$.

We first consider case one with $i \neq j, k \neq l$.

Proposition 2.11 Let $i \neq j, k \neq l$, and $a, b \in \mathbb{C}^{\times}$. We have

1. If $i, j, k, l$ are distinct integers, then $\left[X_{i j}(a, z), X_{k l}(b, w)\right]=0$;

2. If $j=k, i \neq l$, then

$$
\left[X_{i j}(a, z), X_{j l}(b, w)\right]=2 X_{i l}(a b, z) \delta\left(\frac{w}{a z}\right) ;
$$

3. If $j=k, i=l$, and $a b \neq 1$, then

$$
\begin{gathered}
{\left[X_{i j}(a, z), X_{j i}(b, w)\right]=2 X_{i i}(a b, z) \delta\left(\frac{w}{a z}\right)-2 X_{j j}(a b, w) \delta\left(\frac{b w}{z}\right)} \\
+2 \frac{1+a b}{1-a b}\left(\delta\left(\frac{w}{a z}\right)-\delta\left(\frac{b w}{z}\right)\right) ;
\end{gathered}
$$

4. If $j=k, i=l$, and $a b=1$, then

$$
\left[X_{i j}(a, z), X_{j i}(b, w)\right]=2\left(X_{i i}(1, z)-X_{j j}(1, w)\right) \delta\left(\frac{b w}{z}\right)+4(D \delta)\left(\frac{b w}{z}\right) .
$$

Proof. From (2.8), one can easily see the first statement. For the statement two of the proposition, we have

$$
\begin{aligned}
\Delta_{j l}^{i j} & =\varepsilon\left(\epsilon_{i}, \epsilon_{l}\right)\left(\frac{a z+w}{a z}\right)\left[\frac{1}{1-\frac{w}{a z}}+\frac{\frac{a z}{w}}{1-\frac{a z}{w}}\right] \\
& =\varepsilon\left(\epsilon_{i}, \epsilon_{l}\right)\left(\frac{a z+w}{a z}\right) \delta\left(\frac{w}{a z}\right) .
\end{aligned}
$$


Thus, by Lemma 2.10, we get

$$
\begin{aligned}
& {\left[X_{i j}(a, z), X_{j l}(b, w)\right]=\varepsilon\left(\epsilon_{i}, \epsilon_{l}\right)\left(\frac{a z+w}{a z}\right) \delta\left(\frac{w}{a z}\right): X_{i j}(a, z) X_{j l}(b, w):} \\
& =2 \varepsilon\left(\epsilon_{i}, \epsilon_{l}\right) \delta\left(\frac{w}{a z}\right) e^{\overline{\epsilon_{i}-\epsilon_{l}}} E_{-}\left(\epsilon_{i}, z\right) E_{-}\left(-\epsilon_{l}, a b z\right) E_{+}\left(\epsilon_{i}, z\right) E_{+}\left(-\epsilon_{l}, a b z\right) \\
& =2 X_{i l}(a b, z) \delta\left(\frac{w}{a z}\right) .
\end{aligned}
$$

This gives the statement two. Similarly for statement three, we have from (2.8) and Proposition 2.8

$$
\begin{aligned}
\Delta_{j i}^{i j} & =\left(\frac{a z+w}{a z}\right)\left(\frac{z+b w}{z}\right)\left[\frac{1}{1-\frac{w}{a z}} \frac{1}{1-\frac{b w}{z}}-\frac{\frac{a z}{w}}{1-\frac{a z}{w}} \frac{\frac{z}{b w}}{1-\frac{z}{b w}}\right] \\
& =\left(\frac{a z+w}{a z}\right)\left(\frac{z+b w}{z}\right)\left(\frac{1}{1-a b} \delta\left(\frac{w}{a z}\right)+\frac{1}{1-\frac{1}{a b}} \delta\left(\frac{b w}{z}\right)\right) .
\end{aligned}
$$

Thus, from Lemma 2.10, we obtain

$$
\begin{aligned}
& {\left[X_{i j}(a, z), X_{j i}(b, w)\right] } \\
= & \left(\frac{a z+w}{a z}\right)\left(\frac{z+b w}{z}\right)\left(\frac{1}{1-a b} \delta\left(\frac{w}{a z}\right)+\frac{1}{1-\frac{1}{a b}} \delta\left(\frac{b w}{z}\right)\right): X_{i j}(a, z) X_{j i}(b, w): \\
= & 2 \frac{1+a b}{1-a b} \delta\left(\frac{w}{a z}\right) E_{-}\left(\epsilon_{i}, z\right) E_{-}\left(-\epsilon_{i}, a b z\right) E_{+}\left(\epsilon_{i}, z\right) E_{+}\left(-\epsilon_{i}, a b z\right) \\
& +2 \frac{a b+1}{a b-1} \delta\left(\frac{b w}{z}\right) E_{-}\left(\epsilon_{j}, w\right) E_{-}\left(-\epsilon_{j}, a b w\right) E_{+}\left(\epsilon_{j}, w\right) E_{+}\left(-\epsilon_{j}, a b w\right) \\
= & 2 X_{i i}(a b, z) \delta\left(\frac{w}{a z}\right)-2 X_{j j}(a b, w) \delta\left(\frac{b w}{z}\right)+2 \frac{1+a b}{1-a b}\left(\delta\left(\frac{w}{a z}\right)-\delta\left(\frac{b w}{z}\right)\right) .
\end{aligned}
$$

Finally we prove the statement four of the proposition. In this case we have from (2.8)

$$
\begin{aligned}
\Delta_{j i}^{i j} & =\left(\frac{z+b w}{z}\right)^{2}\left[\left(\frac{1}{1-\frac{b w}{z}}\right)^{2}-\left(\frac{\frac{z}{b w}}{1-\frac{z}{b w}}\right)^{2}\right] \\
& =\left(\frac{z+b w}{z}\right)^{2}\left(\frac{b w}{z}\right)^{-1}(D \delta)\left(\frac{b w}{z}\right) .
\end{aligned}
$$

Thus, from this and Lemma 2.10, we obtain

$$
\begin{aligned}
& {\left[X_{i j}(a, z), X_{j i}(b, w)\right] } \\
= & \left(\frac{z+b w}{z}\right)^{2}\left(\frac{b w}{z}\right)^{-1}(D \delta)\left(\frac{b w}{z}\right): X_{i j}(a, z) X_{j i}(b, w): \\
= & 4(D \delta)\left(\frac{b w}{z}\right)+4 \delta\left(\frac{b w}{z}\right)\left(2 \epsilon_{i}(z)-2 \epsilon_{j}(a z)\right) \\
= & 2\left(X_{i i}(1, z)-X_{j j}(1, w)\right) \delta\left(\frac{b w}{z}\right)+4(D \delta)\left(\frac{b w}{z}\right) .
\end{aligned}
$$

This completes the proof of the Proposition. 
Now we consider case two for $\left[X_{i j}(a, z), X_{k l}(b, w)\right]$ with $i=j, k \neq l$. The result will be divided into three subcases.

Proposition 2.12 Let $1 \leq i, k, j \leq \nu$, and $a, b \in \mathbb{C}^{\times}$. We have

1. If $i, k, j$ are distinct, then $\left[X_{i i}(a, z), X_{k j}(b, w)\right]=0$;

2. If $i \neq j$, and $a=1$, then

$$
\left[X_{i i}(a, z), X_{i j}(b, w)\right]=2 X_{i j}(b, w)\left(\delta\left(\frac{w}{z}\right)-\delta\left(-\frac{w}{z}\right)\right) ;
$$

3. If $i \neq j$, and $a \neq 1$, then

$$
\left[X_{i i}(a, z), X_{i j}(b, w)\right]=2 X_{i j}(a b, z) \delta\left(\frac{w}{a z}\right)+2 X_{j i}\left(a b^{-1}, b z\right) \delta\left(-\frac{w}{z}\right) .
$$

Proof. The statement one of the proposition is clear. To prove the second one, we have

$$
\begin{aligned}
& {\left[X_{i i}(a, z), X_{i j}(b, w)\right]=\left[4 \epsilon_{i}(z), X_{i j}(b, w)\right] } \\
= & 4\left[\epsilon_{i}(z), \varepsilon\left(\epsilon_{i}, \epsilon_{j}\right) e^{\overline{\epsilon_{i}-\epsilon_{j}}} E_{-}\left(\epsilon_{i}, w\right) E_{-}\left(-\epsilon_{j}, b w\right) E_{+}\left(\epsilon_{i}, w\right) E_{+}\left(-\epsilon_{j}, b w\right)\right] \\
= & 4 \varepsilon\left(\epsilon_{i}, \epsilon_{j}\right) e^{\overline{\epsilon_{i}-\epsilon_{j}}} E_{-}\left(\epsilon_{i}, w\right) E_{-}\left(-\epsilon_{j}, b w\right) E_{+}\left(\epsilon_{i}, w\right) E_{+}\left(-\epsilon_{j}, b w\right) \sum_{n \in 2 \mathbb{Z}+1}\left(\frac{z}{w}\right)^{n} \\
= & 2 X_{i j}(b, w)\left(\delta\left(\frac{w}{z}\right)-\delta\left(-\frac{w}{z}\right)\right),
\end{aligned}
$$

where in the second last identity we have used Lemma 2.3. We now prove the last part of the proposition. By (2.8), and Proposition 2.8, Lemma 2.10, we have

$$
\begin{aligned}
& {\left[X_{i i}(a, z), X_{i j}(b, w)\right] } \\
= & \frac{1+a}{1-a} \varepsilon\left(\epsilon_{i}, \epsilon_{j}\right) \Delta_{j i}^{i i}: X_{i i}(a, z) X_{i j}(b, w): \\
= & \frac{1+a}{1-a} \varepsilon\left(\epsilon_{i}, \epsilon_{j}\right)\left(1+\frac{w}{a z}\right)\left(1-\frac{w}{z}\right)\left[\frac{1}{1-\frac{w}{a z}} \frac{1}{1+\frac{w}{z}}+\frac{\frac{z}{w}}{1+\frac{z}{w}} \frac{\frac{a z}{w}}{1-\frac{a z}{w}}\right]: X_{i i}(a, z) X_{i j}(b, w): \\
= & \frac{1+a}{1-a} \varepsilon\left(\epsilon_{i}, \epsilon_{j}\right)\left(1+\frac{w}{a z}\right)\left(1-\frac{w}{z}\right)\left[\frac{1}{1+a} \delta\left(\frac{w}{a z}\right)+\frac{1}{1+\frac{1}{a}} \delta\left(-\frac{w}{z}\right)\right]: X_{i i}(a, z) X_{i j}(b, w): \\
= & 2 \varepsilon\left(\epsilon_{i}, \epsilon_{j}\right) e^{\overline{\epsilon_{i}-\epsilon_{j}}} E_{-}\left(\epsilon_{i}, z\right) E_{-}\left(-\epsilon_{j}, a b z\right) E_{+}\left(\epsilon_{i}, z\right) E_{+}\left(-\epsilon_{j}, a b z\right) \delta\left(\frac{w}{a z}\right) \\
& -2 \varepsilon\left(\epsilon_{i}, \epsilon_{j}\right) e^{\overline{\epsilon_{i}-\epsilon_{j}}} E_{-}\left(\epsilon_{i},-a z\right) E_{-}\left(-\epsilon_{j},-b z\right) E_{+}\left(\epsilon_{i},-a z\right) E_{+}\left(-\epsilon_{j},-b z\right) \delta\left(-\frac{w}{z}\right) \\
= & 2 X_{i j}(a b, z) \delta\left(\frac{w}{a z}\right)-2 X_{i j}\left(b a^{-1},-a z\right) \delta\left(-\frac{w}{z}\right) \\
= & 2 X_{i j}(a b, z) \delta\left(\frac{w}{a z}\right)+2 X_{j i}\left(a b^{-1}, b z\right) \delta\left(-\frac{w}{z}\right)
\end{aligned}
$$

Finally we consider case 3 , that is to compute $\left[X_{i j}(a, z), X_{k l}(b, w)\right]$ with $i=j=k=l$, and $a, b \in \mathbb{C}^{\times}$. From the definition of $X_{i i}(a, z), X_{i i}(b, w)$, we may assume that $a \neq-1, b \neq-1$. 
Proposition 2.13 Let $1 \leq i \leq \nu$, and $a, b \in \mathbb{C}^{\times}$. We have

1. For $a=b=1$, we have

$$
\left[X_{i i}(1, z), X_{i i}(1, w)\right]=4\left((D \delta)\left(\frac{w}{z}\right)-(D \delta)\left(-\frac{w}{z}\right)\right)
$$

2. For $a=1, b \neq 1$, then

$$
\begin{gathered}
{\left[X_{i i}(1, z), X_{i i}(b, w)\right]=2 X_{i i}(b, w)\left(\delta\left(\frac{w}{z}\right)-\delta\left(-\frac{w}{z}\right)-\delta\left(\frac{b w}{z}\right)+\delta\left(-\frac{b w}{z}\right)\right)} \\
+2 \frac{1+b}{1-b}\left(\delta\left(\frac{w}{z}\right)-\delta\left(-\frac{w}{z}\right)-\delta\left(\frac{b w}{z}\right)+\delta\left(-\frac{b w}{z}\right)\right) ;
\end{gathered}
$$

3. If $a \neq 1, b \neq 1$, and $a \neq b, a b \neq 1$, then

$$
\begin{aligned}
& {\left[X_{i i}(a, z), X_{i i}(b, w)\right] } \\
= & 2\left(X_{i i}(a b, z)+\frac{1+a b}{1-a b}\right) \delta\left(\frac{w}{a z}\right)-2\left(X_{i i}\left(a b, b^{-1} z\right)+\frac{1+a b}{1-a b}\right) \delta\left(\frac{b w}{z}\right) \\
& +2\left(X_{i i}\left(a b^{-1}, b z\right)+\frac{1+a b^{-1}}{1-a b^{-1}}\right) \delta\left(-\frac{w}{z}\right)-2\left(X_{i i}\left(a b^{-1}, z\right)+\frac{1+a b^{-1}}{1-a b^{-1}}\right) \delta\left(-\frac{b w}{a z}\right) ;
\end{aligned}
$$

4. If $a \neq 1, b \neq 1$, and $a b=1, a \neq b$, then

$$
\begin{aligned}
& {\left[X_{i i}(a, z), X_{i i}\left(a^{-1}, w\right)\right] } \\
= & 2\left(X_{i i}(1, z)-X_{i i}(1, a z)\right) \delta\left(\frac{w}{a z}\right)+4(D \delta)\left(\frac{w}{a z}\right) \\
& +2\left(X_{i i}\left(a^{2}, a^{-1} z\right)+\frac{1+a^{2}}{1-a^{2}}\right) \delta\left(-\frac{w}{z}\right)-2\left(X_{i i}\left(a^{2}, z\right)+\frac{1+a^{2}}{1-a^{2}}\right) \delta\left(-\frac{w}{a^{2} z}\right) ;
\end{aligned}
$$

5. If $a \neq 1, b \neq 1$, and $a=b, a b \neq 1$, then

$$
\begin{aligned}
& {\left[X_{i i}(a, z), X_{i i}(a, w)\right] } \\
= & 2\left(X_{i i}\left(a^{2}, z\right)+\frac{1+a^{2}}{1-a^{2}}\right) \delta\left(\frac{w}{a z}\right)-2\left(X_{i i}\left(a^{2}, a^{-1} z\right)+\frac{1+a^{2}}{1-a^{2}}\right) \delta\left(\frac{a w}{z}\right) \\
& -2\left(X_{i i}(1, a z)-X_{i i}(1, z)\right) \delta\left(-\frac{w}{z}\right)-4(D \delta)\left(-\frac{w}{z}\right) .
\end{aligned}
$$

Proof. The first part of the proposition is straightforward. To prove the second part, we have

$$
\begin{gathered}
{\left[X_{i i}(1, z), X_{i i}(b, w)\right]} \\
=4\left[\epsilon_{i}(z), \frac{1+b}{1-b} E_{-}\left(\epsilon_{i}, w\right) E_{-}\left(-\epsilon_{i}, b w\right) E_{+}\left(\epsilon_{i}, w\right) E_{+}\left(-\epsilon_{i}, b w\right)\right] .
\end{gathered}
$$

Then by Lemma 2.3, we have

$$
\begin{gathered}
{\left[X_{i i}(1, z), X_{i i}(b, w)\right]=2 \frac{1+b}{1-b} E_{-}\left(\epsilon_{i}, w\right) E_{-}\left(-\epsilon_{i}, b w\right) E_{+}\left(\epsilon_{i}, w\right) E_{+}\left(-\epsilon_{i}, b w\right)} \\
\cdot\left(\delta\left(\frac{w}{z}\right)-\delta\left(-\frac{w}{z}\right)-\delta\left(\frac{b w}{z}\right)+\delta\left(-\frac{b w}{z}\right)\right)
\end{gathered}
$$




$$
=2\left(X_{i i}(b, w)+\frac{1+b}{1-b}\right)\left(\delta\left(\frac{w}{z}\right)-\delta\left(-\frac{w}{z}\right)-\delta\left(\frac{b w}{z}\right)+\delta\left(-\frac{b w}{z}\right)\right) .
$$

Next, we prove the third part of the proposition. Note that in this case, one has $1 \neq a^{-1} \neq$ $b \neq a^{-1} b$. Now, from (2.8) and Proposition 2.8, Lemma 2.10, we have

$$
\begin{aligned}
& {\left[X_{i i}(a, z), X_{i i}(b, w)\right] } \\
= & : X_{i i}(a, z) X_{i i}(b, w): \frac{1+a}{1-a} \frac{1+b}{1-b}\left(\frac{a z+w}{a z}\right)\left(\frac{z-w}{z}\right)\left(\frac{a z-b w}{a z}\right)\left(\frac{z+b w}{z}\right) \\
& \cdot\left(\frac{1}{1-\frac{w}{a z}} \frac{1}{1+\frac{w}{z}} \frac{1}{1+\frac{b w}{a z}} \frac{1}{1-\frac{b w}{z}}-\frac{\frac{a z}{w}}{1-\frac{a z}{w}} \frac{\frac{z}{w}}{1+\frac{z}{w}} \frac{\frac{a z}{b w}}{1+\frac{a z}{b w}} \frac{\frac{z}{b w}}{1-\frac{z}{b w}}\right) \\
= & : X_{i i}(a, z) X_{i i}(b, w): \frac{1+a}{1-a} \frac{1+b}{1-b}\left(\frac{a z+w}{a z}\right)\left(\frac{z-w}{z}\right)\left(\frac{a z-b w}{a z}\right)\left(\frac{z+b w}{z}\right) \\
& \cdot\left[\frac{1}{(1-b a)(1+a)(1+b)} \delta\left(\frac{w}{a z}\right)+\frac{1}{\left(1-a^{-1} b^{-1}\right)\left(1+a^{-1}\right)\left(1+b^{-1}\right)} \delta\left(\frac{b w}{z}\right)\right. \\
& \left.+\frac{1}{\left(1-b a^{-1}\right)(1+b)\left(1+a^{-1}\right)} \delta\left(-\frac{w}{z}\right)+\frac{1}{\left(1-b^{-1} a\right)\left(1+b^{-1}\right)(1+a)} \delta\left(-\frac{b w}{a z}\right)\right] \\
= & : X_{i i}(a, z) X_{i i}(b, w):\left[\frac{1+a b}{1-a b} \delta\left(\left(\frac{w}{a z}\right)-\delta\left(\frac{b w}{z}\right)\right)+\frac{1+a b^{-1}}{1-a b^{-1}}\left(\delta\left(-\frac{w}{z}\right)-\delta\left(-\frac{b w}{a z}\right)\right)\right] \\
= & 2\left(X_{i i}(a b, z)+\frac{1+a b}{1-a b}\right) \delta\left(\frac{w}{a z}\right)-2\left(X_{i i}\left(a b, b^{-1} z\right)+\frac{1+a b}{1-a b}\right) \delta\left(\frac{b w}{z}\right) \\
& +2\left(X_{i i}\left(a b^{-1}, b z\right)+\frac{1+a b^{-1}}{1-a b^{-1}}\right) \delta\left(-\frac{w}{z}\right)-2\left(X_{i i}\left(a b^{-1}, z\right)+\frac{1+a b^{-1}}{1-a b^{-1}}\right) \delta\left(-\frac{b w}{a z}\right)
\end{aligned}
$$

Finally, we will prove the last part of proposition and the proof of the fourth part is similar to that of this case, which we omit. From (2.8) and Proposition 2.7, Lemma 2.10, one has

$$
\begin{aligned}
& {\left[X_{i i}(a, z), X_{i i}(a, w)\right] } \\
= & : X_{i i}(a, z) X_{i i}(a, w):\left(\frac{1+a}{1-a}\right)^{2}\left(\frac{a z+w}{a z}\right)\left(\frac{z-w}{z}\right)^{2}\left(\frac{z+a w}{z}\right) \\
& \cdot\left[\frac{1}{1-\frac{w}{a z}}\left(\frac{1}{1+\frac{w}{z}}\right)^{2} \frac{1}{1-\frac{a w}{z}}-\frac{\frac{a z}{w}}{1-\frac{a z}{w}}\left(\frac{\frac{z}{w}}{1+\frac{z}{w}}\right)^{2} \frac{\frac{z}{a w}}{1-\frac{z}{a w}}\right] \\
= & : X_{i i}(a, z) X_{i i}(a, w):\left(\frac{1+a}{1-a}\right)^{2}\left(\frac{a z+w}{a z}\right)\left(\frac{z-w}{z}\right)^{2}\left(\frac{z+a w}{z}\right) \\
& {\left[\frac{1}{(1+a)^{2}\left(1-a^{2}\right)} \delta\left(\frac{w}{a z}\right)+\frac{1}{\left(1+a^{-1}\right)^{2}\left(1-a^{-2}\right)} \delta\left(\frac{a w}{z}\right)\right.} \\
& \left.+\frac{1}{(1+a)\left(1+a^{-1}\right)}\left[(D \delta)\left(-\frac{w}{z}\right)+2 \delta\left(-\frac{w}{z}\right)\right]\right]
\end{aligned}
$$




$$
\begin{aligned}
= & : X_{i i}(a, z) X_{i i}(a, w): \frac{1+a^{2}}{1-a^{2}} \delta\left(\left(\frac{w}{a z}\right)-\delta\left(\frac{a w}{z}\right)\right)-2\left(\epsilon_{i}(a z)-\epsilon_{i}(z)\right) \delta\left(-\frac{w}{z}\right)-4(D \delta)\left(-\frac{w}{z}\right) \\
& +: X_{i i}(a, z) X_{i i}(a, w): \frac{a}{(1-a)^{2}}\left[2\left(\frac{a z+w}{a z}\right)\left(\frac{z-w}{z}\right)^{2}\left(\frac{z+a w}{z}\right)\right. \\
& -\frac{w}{a z}\left(\frac{z-w}{z}\right)^{2}\left(\frac{z+a w}{z}\right)-\frac{a w}{z}\left(\frac{a z+w}{a z}\right)\left(\frac{z-w}{z}\right)^{2} \\
& \left.-2\left(-\frac{w}{z}\right)\left(\frac{a z+w}{a z}\right)\left(\frac{z-w}{z}\right)\left(\frac{z+a w}{z}\right)\right] \delta\left(-\frac{w}{z}\right) \\
= & 2\left(X_{i i}\left(a^{2}, z\right)+\frac{1+a^{2}}{1-a^{2}}\right) \delta\left(\frac{w}{a z}\right)-2\left(X_{i i}\left(a^{2}, a^{-1} z\right)+\frac{1+a^{2}}{1-a^{2}}\right) \delta\left(\frac{a w}{z}\right) \\
& -2\left(X_{i i}(1, a z)-X_{i i}(1, z)\right) \delta\left(-\frac{w}{z}\right)-4(D \delta)\left(-\frac{w}{z}\right) .
\end{aligned}
$$

Remark 2.14 It is easy to see that

$$
\frac{\delta(z)-a^{2} \delta(a z)}{1-a} \rightarrow(D \delta)(z)+2 \delta(z)
$$

as $a \rightarrow 1$. This gives the following identities

$$
\frac{\delta(w / a z)-a^{2} b^{2} \delta(b w / z)}{1-a b} \rightarrow(D \delta)(w / a z)+2 \delta(w / a z)
$$

as $a b \rightarrow 1$, and

$$
\frac{a^{2} \delta(-w / z)-b^{2} \delta(-b w / a z)}{a-b} \rightarrow a(D \delta)(-w / z)+2 a \delta(-w / z)
$$

as $b \rightarrow a$.

In summary, from Proposition 2.11, 2.12 and 2.13, we can get the following commutator relations

Theorem 2.15 For $1 \leq i, j, k, l \leq \nu$ and $a, b \in \mathbb{C}^{\times}$, one has

$$
\begin{aligned}
& {\left[X_{i j}(a, z), X_{k l}(b, w)\right] } \\
= & 2 \delta_{j k}\left(X_{i l}(a b, z)+\delta_{i l}\left(1-\delta_{a b, 1}\right) \frac{1+a b}{1-a b}\right) \delta\left(\frac{w}{a z}\right) \\
- & 2 \delta_{i l}\left(X_{k j}\left(a b, b^{-1} z\right)+\delta_{j k}\left(1-\delta_{a b, 1}\right) \frac{1+a b}{1-a b}\right) \delta\left(\frac{b w}{z}\right) \\
+ & 2 \delta_{i k}\left(X_{l j}\left(a b^{-1}, b z\right)+\delta_{j l}\left(1-\delta_{a, b}\right) \frac{1+a b^{-1}}{1-a b^{-1}}\right) \delta\left(-\frac{w}{z}\right) \\
- & 2 \delta_{j l}\left(X_{i k}\left(a b^{-1}, z\right)+\delta_{i k}\left(1-\delta_{a, b}\right) \frac{1+a b^{-1}}{1-a b^{-1}}\right) \delta\left(-\frac{b w}{a z}\right) \\
+ & 4 \delta_{i l} \delta_{j k} \delta_{a b, 1}(D \delta)\left(\frac{w}{a z}\right)-4 \delta_{i k} \delta_{j l} \delta_{a, b}(D \delta)\left(-\frac{w}{z}\right) .
\end{aligned}
$$




\section{Representations of unitary Lie algebras}

In this section we begin by recalling the unitary Lie algebra $\mathfrak{u}_{\nu}\left(\mathcal{R}_{\sigma}{ }^{-}\right)$associated with a skew Laurent polynomial ring $\mathcal{R}_{\sigma}$ and an anti-involution ${ }^{-}$, which was first introduced in [AF]. Using the commutator relation among the vertex operators developed in Theorem 2.15, we find that $M$ turn to a representation for a non-trivial central extension of the unitary Lie algebra $\mathfrak{u}_{\nu}\left(\mathcal{R}_{\sigma},{ }^{-}\right)$. Moreover, we determine the irreducible decomposition of $M$ explicitly.

Let $G$ be an abelian group with a character $\sigma$, that is, $\sigma: G \rightarrow \mathbb{C}^{\times}$is a group homomorphism. Extend $\sigma$, still call $\sigma$, to be an automorphism of the group algebra $\mathcal{R}$ associated to $G$ determined by $\sigma\left(e^{\alpha}\right)=\sigma(\alpha) e^{\alpha}, \alpha \in G$. Then we can form the skew Laurent polynomial ring $\mathcal{R}_{\sigma}=\mathcal{R}\left[t^{ \pm 1}, \sigma\right]$ with basis $t^{m} e^{\alpha}, m \in \mathbb{Z}, \alpha \in G$ and multiplication

$$
e^{\alpha} t^{m}=(\sigma(\alpha))^{m} t^{m} e^{\alpha}
$$

For simplicity, we denote $\sigma(\alpha)$ by $\tilde{\alpha}$ in the following.

Let ${ }^{-}$be an anti-involution of $\mathcal{R}_{\sigma}$ defined by

$$
\bar{t}=-t, \overline{e^{\alpha}}=e^{-\alpha}
$$

then $\overline{t^{m} e^{\alpha}}=(-\tilde{\alpha})^{-m} t^{m} e^{-\alpha}$ for $\alpha \in G, m \in \mathbb{Z}$.

Define an operator on the $\nu \times \nu$ matrix algebra $M_{\nu}\left(\mathcal{R}_{\sigma}\right)$,

$$
\begin{aligned}
{ }^{*}: M_{\nu}\left(\mathcal{R}_{\sigma}\right) & \rightarrow M_{\nu}\left(\mathcal{R}_{\sigma}\right), \\
X & \mapsto \bar{X}^{t}
\end{aligned}
$$

where $X \in M_{\nu}\left(\mathcal{R}_{\sigma}\right)$ and $X^{t}$ is the transpose of the matrix $X$. Then $\theta(X)=-X^{*}$ induces an involution on the Lie algebra $g l_{\nu}\left(\mathcal{R}_{\sigma}\right)=M_{\mu}\left(\mathcal{R}_{\sigma}\right)^{-}$. The fixed point subalgebra of $\theta$ is called a unitary Lie algebra (see $[\mathrm{AF}],[\mathrm{G} 2])$ :

$$
\mathfrak{u}_{\nu}\left(\mathcal{R}_{\sigma}{ }^{-}\right)=\left\{X \in M_{\nu}\left(\mathcal{R}_{\sigma}\right) \mid X^{*}=-X\right\}
$$

For convenience, we set

$$
e_{i j}(m, \alpha)=E_{i j} t^{m} e^{\alpha}-E_{j i} \overline{t^{m} e^{\alpha}}
$$

where $1 \leq i, j \leq \nu, \alpha \in G, m \in \mathbb{Z}$. Then $e_{i j}(m, \alpha)=-(-\tilde{\alpha})^{-m} e_{j i}(m,-\alpha)$ and $\mathfrak{u}_{\nu}\left(\mathcal{R}_{\sigma}{ }^{-}{ }^{-}\right)$is spanned by $e_{i j}(m, \alpha)$ for all $1 \leq i, j \leq \nu, m \in \mathbb{Z}$ and $\alpha \in G$.

We define a 1-dimensional central extension of $\widehat{g l}_{\nu}\left(\mathcal{R}_{\sigma}\right)=g l_{\nu}\left(\mathcal{R}_{\sigma}\right) \oplus \mathbb{C} c$ of $g l_{\nu}\left(\mathcal{R}_{\sigma}\right)$ with Lie bracket (See $[\mathrm{G} 3,4])$

$$
\begin{aligned}
& {\left[E_{i j} t^{m} e^{\alpha}, E_{k l} t^{n} e^{\beta}\right]=\delta_{j k} \tilde{\alpha}^{n} E_{i l} t^{m+n} e^{\alpha+\beta}} \\
& -\delta_{i l} \tilde{\beta}^{m} E_{k j} t^{m+n} e^{\alpha+\beta}+m \delta_{i l} \delta_{j k} \delta_{m+n, 0} \delta_{\tilde{\alpha} \tilde{\beta}, 1} \tilde{\alpha}^{n} c
\end{aligned}
$$

where $1 \leq i, j, k, l \leq \nu, m, n \in \mathbb{Z}$ and $\alpha, \beta \in G$.

Now we can from a central extension of $\mathfrak{u}_{\nu}\left(\mathcal{R}_{\sigma},{ }^{-}\right)$

$$
\widehat{\mathfrak{u}}_{\nu}\left(\mathcal{R}_{\sigma},{ }^{-}\right)=\mathfrak{u}_{\nu}\left(\mathcal{R}_{\sigma},{ }^{-}\right) \oplus \mathbb{C} c
$$


with Lie bracket as (3.1). Set

$$
e_{i j}(\alpha, z)=\sum_{n \in \mathbb{Z}} e_{i j}(n, \alpha) z^{-n},
$$

for $1 \leq i, j \leq \nu, \alpha \in G$. Then we have

Proposition 3.1 In $\widehat{\mathfrak{u}}_{\nu}\left(\mathcal{R}_{\sigma},{ }^{-}\right)$,

$$
\begin{aligned}
& {\left[e_{i j}(\alpha, z), e_{k l}(\beta, w)\right] } \\
= & \delta_{j k} e_{i l}(\alpha+\beta, z) \delta\left(\frac{w}{\tilde{\alpha} z}\right)-\delta_{i l} e_{k j}\left(\alpha+\beta, \tilde{\beta}^{-1} z\right) \delta\left(\frac{\tilde{\beta} w}{z}\right) \\
+ & \delta_{i k} e_{l j}(\alpha-\beta, \tilde{\beta} z) \delta\left(-\frac{w}{z}\right)-\delta_{j l} e_{i k}(\alpha-\beta, z) \delta\left(-\frac{\tilde{\beta} w}{\tilde{\alpha} z}\right) \\
+ & 2 \delta_{i l} \delta_{j k} \delta_{\tilde{\alpha} \tilde{\beta}, 1} c(D \delta)\left(\frac{w}{\tilde{\alpha} z}\right)-2 \delta_{i k} \delta_{j l} \delta_{\tilde{\alpha}, \tilde{\beta}} c(D \delta)\left(-\frac{w}{z}\right) .
\end{aligned}
$$

where $1 \leq i, j, k, l \leq \nu, \alpha, \beta \in G$.

Proof. We have

$$
\begin{aligned}
& {\left[\sum_{m \in \mathbb{Z}}\left(E_{i j} t^{m} e^{\alpha}-E_{j i} \overline{t^{m} e^{\alpha}}\right) z^{-m}, \sum_{n \in \mathbb{Z}}\left(E_{k l} t^{n} e^{\beta}-E_{l k} \overline{t^{n} e^{\beta}}\right) w^{-n}\right] } \\
= & \sum_{m, n \in \mathbb{Z}} \delta_{j k}\left(E_{i l} t^{m} e^{\alpha} t^{n} e^{\beta}-E_{l i} \overline{t^{n} e^{\beta}} \overline{t^{m} e^{\alpha}}\right) z^{-m} w^{-n} \\
& -\sum_{m, n \in \mathbb{Z}} \delta_{i l}\left(E_{k j} t^{n} e^{\beta} t^{m} e^{\alpha}-E_{j k} \overline{t^{m} e^{\alpha} t^{n} e^{\beta}}\right) z^{-m} w^{-n} \\
+ & \sum_{m, n \in \mathbb{Z}} \delta_{i k}\left(E_{j l} \overline{t^{n} e^{\beta}} t^{m} e^{\alpha}-E_{l j} \overline{t^{m} e^{\alpha}} t^{n} e^{\beta}\right) z^{-m} w^{-n} \\
& -\sum_{m, n \in \mathbb{Z}} \delta_{j l}\left(E_{i k} t^{m} e^{\alpha} \overline{t^{n} e^{\beta}}-E_{k i} t^{n} e^{\beta} \overline{t^{m} e^{\alpha}}\right) z^{-m} w^{-n} \\
+ & \sum_{m \in \mathbb{Z}} 2 m \delta_{j k} \delta_{i l} \delta_{\tilde{\alpha} \tilde{\beta}, 1}(\tilde{\alpha})^{-m} c z^{-m} w^{m}-\sum_{m \in \mathbb{Z}} 2 m \delta_{i k} \delta_{j l} \delta_{\tilde{\alpha}, \tilde{\beta}} c(-z)^{-m} w^{m} \\
= & \sum_{m, n \in \mathbb{Z}} \delta_{j k} e_{i l}(m+n, \alpha+\beta) z^{-m-n}(\tilde{\alpha} z)^{n} w^{-n} \\
& -\sum_{m, n \in \mathbb{Z}} \delta_{i l} e_{k j}(m+n, \alpha+\beta)(\tilde{\beta}-1 z)^{-m-n} z^{n}(\tilde{\beta} w)^{-n} \\
+ & \sum_{m, n \in \mathbb{Z}} \delta_{i k} e_{l j}(m+n, \alpha-\beta)(\tilde{\beta} z)^{-m-n} z^{n}(-w)^{-n} \\
& -\sum_{m, n \in \mathbb{Z}} \delta_{j l} e_{i k}(m+n, \alpha-\beta) z^{-m-n}(\tilde{\alpha} z)^{n}(-\tilde{\beta} w)^{-n} \\
+ & 2 \delta_{i l} \delta_{j k} \delta_{\tilde{\alpha} \tilde{\beta}, 1} c(D \delta)\left(\frac{w}{\tilde{\alpha} z}\right)-2 \delta_{i k} \delta_{j l} \delta_{\tilde{\alpha}, \tilde{\beta}} c(D \delta)\left(-\frac{w}{z}\right), \\
&
\end{aligned}
$$

as required.

Comparing Theorem 2.15 and Proposition 3.1, one can see that the following result holds true. 
Theorem 3.2 Let $G$ be an abelian group with a character $\sigma$, then $M$ is a vertex operator representation for the unitary Lie algebra $\widehat{\mathfrak{u}}_{\nu}\left(\mathcal{R}_{\sigma},{ }^{-}\right)$with the action given by

$$
2 e_{i j}(m, \alpha) \mapsto x_{i j}(\tilde{\alpha}, m)+\delta_{i j}\left(1-\delta_{\tilde{\alpha}, 1}\right) \frac{1+\tilde{\alpha}}{1-\tilde{\alpha}}, c \mapsto \frac{1}{2},
$$

where $1 \leq i, j \leq \nu$ and $\alpha \in G$.

Next, we are going to determine the irreducible decomposition of $\widehat{\mathfrak{u}}_{\nu}\left(\mathcal{R}_{\sigma},{ }^{-}\right)$-module $M$. Let $\mathbb{C}[\bar{Q}]$ be the subalgebra of $\mathbb{C}[\bar{\Gamma}]$ generated by $e^{\overline{\epsilon_{j}-\epsilon_{j+1}}}, 1 \leq j \leq \nu-1$ which has dimension $2^{\nu-1}$. Using the standard techniques developed in the vertex representation of affine Lie algebras, it is easy to see that if $U$ is an irreducible $\mathbb{C}[\bar{Q}]$-submodule of $\mathbb{C}[\bar{\Gamma}]$, then $U \otimes \mathcal{S}\left(\mathcal{H}^{-}\right)$ remains irreducible as a $\widehat{\mathfrak{u}}_{\nu}\left(\mathcal{R}_{\sigma},{ }^{-}\right)$-module. Therefore, we need to determine the irreducible components of $\mathbb{C}[\bar{\Gamma}]$ for detail. As a by-product, we also give the irreducible decomposition of $\widehat{\mathfrak{u}}_{\nu}\left(\mathcal{R}_{\sigma}{ }^{-}\right)$-module $M$.

We observe that $e^{\bar{\epsilon}_{j}} e^{\bar{\epsilon}_{k}}+e^{\bar{\epsilon}_{k}} e^{\bar{\epsilon}_{j}}=2 \delta_{j k}, 1 \leq j, k \leq \nu$. So, consider the Clifford algebra $C l(\nu)$ with generators $\psi_{j}, 1 \leq j \leq \nu$ and relation

$$
\psi_{j} \psi_{k}+\psi_{k} \psi_{j}=2 \delta_{j k}, 1 \leq j, k \leq \nu .
$$

Since $\operatorname{dim}(\mathbb{C}[\bar{\Gamma}])=\operatorname{dim}(C l(\nu))=2^{\nu}, \mathbb{C}[\bar{\Gamma}]$ is isomorphic to $C l(\nu)$ as associative algebra. Then $\mathbb{C}[\bar{Q}]$ is isomorphic to the subspace of $C l(\nu)$ spanned by products of an even number of elements $\psi_{j}, j=1, \cdots, \nu$.

By the classical representation theory for Clifford algebra, we see that $\mathbb{C}[\bar{Q}]$ is semisimple. Moreover, if $\nu=2 d+1, \mathbb{C}[\bar{Q}]$ has a unique simple module $S$, which has dimension $2^{d}$. If $\nu=2 d, \mathbb{C}[\bar{Q}]$ has exactly two simple module $S^{ \pm}$, which has dimension $2^{d-1}$.

Remark 3.3 It is well-known that the subspace $\mathrm{Cl}_{2}(\nu)$ of $C l(\nu)$ spanned by products of 2 different element $\psi_{i}, i=1, \cdots, \nu$ is closed under the Lie bracket and is isomorphic to the simple orthogonal Lie algebra $\mathfrak{o}(\nu)$. Fix bases in the root systems of types $D_{d}$ and $B_{d}$ as follows:

$$
\begin{aligned}
& \Pi\left(D_{d}\right)=\left\{h_{1}-h_{2}, \cdots, h_{d-1}-h_{d}, h_{d-1}-h_{d}\right\}, \\
& \Pi\left(B_{d}\right)=\left\{h_{1}-h_{2}, \cdots, h_{d-1}-h_{d}, h_{d}\right\} .
\end{aligned}
$$

Then $S$ is the spinor representation of $\mathfrak{o}(2 d+1)$ with highest weight $\frac{1}{2}\left(h_{1}+\cdots+h_{d}\right)$. And $S^{ \pm}$are the semispinor representation of $\mathfrak{o}(2 d)$ with highest weight $\frac{1}{2}\left(h_{1}+\cdots+h_{d-1} \pm h_{d}\right)$.

For $\rho= \pm 1$, let $w_{2 j-1}(\rho)=1+\sqrt{-1} \rho e^{\bar{\epsilon}_{2 j-1}}$ and $w_{2 j}(\rho)=1+\rho e^{\bar{\epsilon}_{2 j}}$. For $\rho_{1}, \cdots, \rho_{\nu} \in\{ \pm 1\}$, we put

$$
w\left(\rho_{1}, \cdots, \rho_{\nu}\right):=\prod_{j=1}^{\nu} w_{j}\left(\rho_{j}\right) .
$$

Then the collection of these elements $\left\{w\left(\rho_{1}, \cdots, \rho_{\nu}\right): \rho_{1}, \cdots, \rho_{\nu}= \pm 1\right\}$ form a basis of $\mathbb{C}[\bar{\Gamma}]$. The construction of such basis elements are motivated by the work [W]. It is easy to check that (or see Lemma $4.1[\mathrm{~W}]$ ) 
Lemma 3.4 For $\rho_{1}, \cdots, \rho_{\nu} \in\{ \pm 1\}$ and $1 \leq j \leq \nu$, we have

$$
e^{\bar{\epsilon}_{j}} w\left(\rho_{1}, \cdots, \rho_{\nu}\right)= \begin{cases}\sqrt{-1} \rho_{j} w\left(-\rho_{1}, \cdots,-\rho_{j}, \rho_{j+1}, \cdots, \rho_{\nu}\right), & \text { if } j \text { is odd } \\ \rho_{j} w\left(-\rho_{1}, \cdots,-\rho_{j-1}, \rho_{j}, \cdots, \rho_{\nu}\right), & \text { if } j \text { is even. }\end{cases}
$$

Then for $1 \leq j \leq\left[\frac{\nu}{2}\right]$, we have

$$
\begin{aligned}
& e^{\overline{\epsilon_{2 j-1}-\epsilon_{2 j}}} w\left(\rho_{1}, \cdots, \rho_{\nu}\right)=-\sqrt{-1} \rho_{2 j-1} \rho_{2 j} w\left(\rho_{1}, \cdots, \rho-\nu\right), \\
& e^{\overline{\epsilon_{2 j}-\epsilon_{2 j+1}}} w\left(\rho_{1}, \cdots, \rho_{\nu}\right)=-\sqrt{-1} \rho_{2 j} \rho_{2 j+1} w\left(\rho_{1}, \cdots, \rho_{2 j-1},-\rho_{2 j},-\rho_{2 j+1}, \rho_{2 j+2}, \cdots, \rho_{\nu}\right) .
\end{aligned}
$$

From (3.3), we see that the signs $\rho_{1}, \rho_{2 j} \rho_{2 j+1}, 1 \leq j \leq\left[\frac{\nu}{2}\right]$ and $\rho_{\nu}$ if $\nu$ is even are invariant under the action of $\mathbb{C}[\bar{Q}]$.

In the case $\nu=2 d+1$, for any tuple $\gamma=\left(\gamma_{1}, \cdots, \gamma_{d+1}\right) \in \mathbb{Z}_{2}^{d+1}, \mathbb{Z}_{2}=\{ \pm 1\}$, set

$$
V_{\nu}(\gamma)=\operatorname{span}_{\mathbb{C}}\left\{w\left(\rho_{1}, \cdots, \rho_{\nu}\right) \mid \rho_{1}=\gamma_{1}, \rho_{2 j} \rho_{2 j+1}=\gamma_{j+1}, 1 \leq j \leq d\right\}
$$

Then $V_{\nu}(\gamma)$ is a $\mathbb{C}[\bar{Q}]$-module with dimension $2^{d}$, hence is simple. So, we have shown that

$$
\mathbb{C}[\bar{\Gamma}]=\oplus_{\gamma \in \mathbb{Z}_{2}^{d+1}} V_{\nu}(\gamma)
$$

where each $V_{\nu}(\gamma)$ is an irreducible $\mathbb{C}[\bar{Q}]$-module.

In the case $\nu=2 d$, for any tuple $\gamma=\left(\gamma_{1}, \cdots, \gamma_{d+1}\right) \in \mathbb{Z}_{2}^{d+1}$, set

$$
V_{\nu}(\gamma)=\operatorname{span}_{\mathbb{C}}\left\{w\left(\rho_{1}, \cdots, \rho_{\nu}\right) \mid \rho_{1}=\gamma_{1}, \rho_{2 j} \rho_{2 j+1}=\gamma_{j+1}, 1 \leq j \leq d-1, \rho_{2 d}=\gamma_{d+1}\right\}
$$

Then $V_{\nu}(\gamma)$ is an irreducible $\mathbb{C}[\bar{Q}]$-module with

$$
\mathbb{C}[\bar{\Gamma}]=\oplus_{\gamma \in \mathbb{Z}_{2}^{d+1}} V_{\nu}(\gamma)
$$

In summary, we have obtained

Theorem 3.5 The $\widehat{\mathfrak{u}}_{\nu}\left(\mathcal{R}_{\sigma},{ }^{-}\right)$-module $M$ is completely reducible. Moreover,

$$
M=\oplus_{\gamma \in \mathbb{Z}_{2}^{\left[\frac{\nu}{2}\right]+1}} V_{\nu}(\gamma) \otimes \mathcal{S}\left(\mathcal{H}^{-}\right),
$$

where each submodule $V_{\nu}(\gamma) \otimes \mathcal{S}\left(\mathcal{H}^{-}\right)$is irreducible as $\widehat{\mathfrak{u}}_{\nu}\left(\mathcal{R}_{\sigma},{ }^{-}\right)$-module and $V_{\nu}(\gamma)$ are defined in (3.4) and (3.5).

\section{Application to elementary unitary Lie algebras}

Let $G$ be an abelian group with a character $\sigma$ and $|\sigma|$ be the order of $\sigma(G)$. Define the set $\Lambda_{\sigma}=\{(m, \alpha) \in(\mathbb{Z}, G)|m \in| \sigma \mid \mathbb{Z}, \alpha \in \operatorname{ker}(\sigma)\}$. Here, we set $|\sigma| \mathbb{Z}=0$ if $|\sigma|=\infty$. Then we have the following basic result about $\mathcal{R}_{\sigma}$ whose proof is straightforward. 
Lemma 4.1 The derived algebra $\mathcal{R}_{\sigma}^{\prime}$ of $\mathcal{R}_{\sigma}$ has a basis consisting of monomials $t^{m} e^{\alpha}$ for $(m, \alpha) \notin \Lambda_{\sigma}$ and the center $Z_{\sigma}$ of $\mathcal{R}_{\sigma}$ has basis consisting of monomials $t^{m} e^{\alpha}$ for $(m, \alpha) \in \Lambda_{\sigma}$. Therefore, we have $\mathcal{R}_{\sigma}=\mathcal{R}_{\sigma}^{\prime} \oplus Z_{\sigma}$.

The elementary unitary Lie algebra $\mathfrak{e u}_{\nu}\left(\mathcal{R}_{\sigma},{ }^{-}\right)$is the derived algebra of $\mathfrak{u}_{\nu}\left(\mathcal{R}_{\sigma}{ }^{-}\right)$which is generated by $e_{i j}(m, \alpha)$ for $1 \leq i \neq j \leq \nu, m \in \mathbb{Z}$ and $\alpha \in G$. By applying Lemma 4.1, one can see that

$$
\mathfrak{u}_{\nu}\left(\mathcal{R}_{\sigma},{ }^{-}\right)=\mathfrak{e u}_{\nu}\left(\mathcal{R}_{\sigma},{ }^{-}\right) \oplus \sum_{(n, \beta) \in \Lambda_{\sigma}} \mathbb{C}\left(\sum_{i=1}^{\nu} e_{i i}(n, \beta)\right) .
$$

In particular, we have that $e_{i i}(m, \alpha)-e_{j j}(m, \alpha), e_{i i}(n, \beta) \in \mathfrak{e u}_{\nu}\left(\mathcal{R}_{\sigma}{ }^{-}\right)$for $1 \leq i \neq j \leq \nu, m, n \in$ $\mathbb{Z}, \alpha, \beta \in G$ and $(n, \beta) \notin \Lambda_{\sigma}$. Let $\widehat{\mathfrak{e u}}_{\nu}\left(\mathcal{R}_{\sigma},{ }^{-}\right)$be the subalgebra of $\widehat{\mathfrak{u}}_{\nu}\left(\mathcal{R}_{\sigma},{ }^{-}\right)$generated by $e_{i j}(m, \alpha)$ for $1 \leq i \neq j \leq \nu, m \in \mathbb{Z}, \alpha \in G$. Then, $\widehat{\mathfrak{e u}}_{\nu}\left(\mathcal{R}_{\sigma}{ }^{-}\right)$is a one-dimensional non-trivial central extension of $\mathfrak{e u}_{\nu}\left(\mathcal{R}_{\sigma},{ }^{-}\right)$.

Remark 4.2 It was known that both $\mathfrak{e u}_{\nu}\left(\mathcal{R}_{\sigma},{ }^{-}\right)$and $\widehat{\mathfrak{e u}}_{\nu}\left(\mathcal{R}_{\sigma}{ }^{-}\right)$are $B C_{d^{-}}$graded with grading subalgebra $\mathfrak{o}(\nu)$ (See $[\mathrm{ABG}]$ for details), where $d=\left[\frac{\nu}{2}\right]$.

Next, we turn to consider the unitarizability of $M$. For our purpose, we may assume that all the elements in $\sigma(G)$ lie in the unity circle in the complex plane, that is, $|a|=1, \forall a \in \sigma(G)$. Define a conjugate linear map $\tau: \widehat{\mathfrak{u}}_{\nu}\left(\mathcal{R}_{\sigma},{ }^{-}\right) \rightarrow \widehat{\mathfrak{u}}_{\nu}\left(\mathcal{R}_{\sigma}{ }^{-}\right)$by letting

$$
\tau\left(e_{i j}(m, \alpha)\right)=(-1)^{(m+1)} e_{i j}(-m, \alpha), \tau(c)=c,
$$

where $1 \leq i, j \leq \nu, m \in \mathbb{Z}$ and $\alpha \in G$. Due to $|\tilde{\alpha}|=1, \alpha \in G$, one can get that $\tau$ is a conjugate anti-involution of $\widehat{\mathfrak{u}}_{\nu}\left(\mathcal{R}_{\sigma},{ }^{-}\right)$.

Define a Hermitian form $<\mid>$ on $\mathcal{H}^{-}$by

$$
<\alpha(m) \mid \beta(n)>=\frac{m}{2}(\alpha, \beta) \delta_{m, n}, \forall \alpha(m), \beta(n) \in \mathcal{H}^{-},
$$

and extend this form to $S\left(\mathcal{H}^{-}\right)$as usual:

$$
<x_{1} \cdots x_{m}\left|y_{1} \cdots y_{n}>=\delta_{m, n} \sum_{\sigma \in S_{m}} \prod_{i=1}^{m}<x_{i}\right| y_{\sigma(i)}>.
$$

Next, we define $<\mid>$ on $\mathbb{C}[\bar{\Gamma}]$ by

$$
<e^{\bar{\alpha}} \mid e^{\bar{\beta}}>=\delta_{\bar{\alpha}, \bar{\beta}}, \forall \alpha, \beta \in \Gamma .
$$

This gives a positive definite Hermitian form on $M$ (See also [FK] (2.12)-(2.14)) such that

$$
\begin{aligned}
<\epsilon_{i}(m) \cdot w \mid v> & =<w \mid \epsilon_{i}(-m) \cdot v> \\
<e^{\bar{\epsilon}_{i}} \cdot w \mid v> & =<w \mid e^{\bar{\epsilon}_{i}} \cdot v>
\end{aligned}
$$

for $1 \leq i \leq \nu, m \in 2 \mathbb{Z}+1, w, v \in M$. 
Proposition 4.3 Suppose that all the elements in $\sigma(G)$ lie in the unity circle in the complex plane, then the $\widehat{\mathfrak{u}}_{\nu}\left(\mathcal{R}_{\sigma},{ }^{-}\right)$-module $M$ is unitary with respect to $\tau$.

Proof. It is sufficient to show that

$$
<X_{i j}(a, z) \cdot w|v>=<w|-X_{i j}\left(a,-z^{-1}\right) \cdot v>
$$

for $1 \leq i, j \leq \nu, a \in \sigma(G), w, v \in M$.

By applying the fact that $\bar{a}=a^{-1}$, one may get that

$$
<\frac{\epsilon_{i}(m)}{m}(a z)^{-m} \cdot w|v>=<w| \frac{-\epsilon_{i}(-m)}{-m}\left(a z^{-1}\right)^{m} \cdot v>,
$$

and that

$$
<E_{ \pm}\left(\epsilon_{i}, a z\right) \cdot w|v>=<w| E_{\mp}\left(-\epsilon_{i}, a z^{-1}\right) \cdot v>=<w \mid E_{\mp}\left(\epsilon_{i},-a z^{-1}\right) \cdot v>
$$

for $1 \leq i \leq \nu, a \in \sigma(G), w, v \in M$.

Hence, for $i \neq j$, we find that

$$
\begin{aligned}
& <X_{i j}(a, z) \cdot w \mid v> \\
= & <w \mid E_{-}\left(-\epsilon_{j},-a z^{-1}\right) E_{-}\left(\epsilon_{i},-z^{-1}\right) E_{+}\left(-\epsilon_{j},-a z^{-1}\right) E_{+}\left(\epsilon_{i},-a z^{-1}\right) e^{\overline{\epsilon_{j}}} e^{\overline{\epsilon_{i}}} \cdot v> \\
= & <w \mid-X_{i j}\left(a,-z^{-1}\right) \cdot v>.
\end{aligned}
$$

The case for $i=j, a=1$ is clear. For the case $i=j, a \neq 1$, one only needs to note that $\overline{\left(\frac{1+a}{1-a}\right)}=-\frac{1+a}{1-a}$.

By taking restriction, we know that each component $V_{\nu}(\gamma) \otimes S\left(\mathcal{H}^{-}\right), \gamma \in \mathbb{Z}_{2}^{\left[\frac{\nu}{2}\right]+1}$ of $M$ is an $\widehat{\mathfrak{e u}}_{\nu}\left(\mathcal{R}_{\sigma}{ }^{-}\right)$-module. Moreover,

Theorem 4.4 Let $V_{\nu}(\gamma) \otimes S\left(\mathcal{H}^{-}\right)$be the $\widehat{\mathfrak{u}}_{\nu}\left(\mathcal{R}_{\sigma},{ }^{-}\right)$-module described in Theorem 3.5,

(1) If $|\sigma|=\infty$ or $|\sigma| \in 2 \mathbb{N}$, then $V_{\nu}(\gamma) \otimes S\left(\mathcal{H}^{-}\right)$is irreducible as $\widehat{\mathfrak{e u}}_{\nu}\left(\mathcal{R}_{\sigma},{ }^{-}\right)$-module.

(2) If $|\sigma|=N \in 2 \mathbb{N}+1$, let $\mathcal{H}_{0}^{-}$be the subspace of $\mathcal{H}^{-}$spanned by $\epsilon_{i}(m)-\epsilon_{j}(m), \epsilon_{i}(n)$ for $1 \leq i \neq j \leq \nu, m, n \in-(2 \mathbb{N}+1), n \notin N \mathbb{Z}$, then $V_{\nu}(\gamma) \otimes S\left(\mathcal{H}_{0}^{-}\right)$is an irreducible $\widehat{\mathfrak{e u}}_{\nu}\left(\mathcal{R}_{\sigma},{ }^{-}\right)$-submodule of $V_{\nu}(\gamma) \otimes S\left(\mathcal{H}^{-}\right)$. Moreover, $V_{\nu}(\gamma) \otimes S\left(\mathcal{H}^{-}\right)$is completely reducible as $\widehat{\mathfrak{e u}}_{\nu}\left(\mathcal{R}_{\sigma},{ }^{-}\right)$-module.

Proof. First, we write

$$
L=\widehat{\mathfrak{u}}_{\nu}\left(\mathcal{R}_{\sigma}{ }^{-}\right), V=V_{\nu}(\gamma) \otimes S\left(\mathcal{H}^{-}\right), V_{0}=V_{\nu}(\gamma) \otimes S\left(\mathcal{H}_{0}^{-}\right),
$$

for simplicity.

If $|\sigma|=\infty$ or $|\sigma| \in 2 \mathbb{N}$, then $e_{i i}(m, 0) \in L, 1 \leq i \leq \nu, m \in 2 \mathbb{Z}+1$ and hence $V$ remains irreducible as $L$-module.

Next, if $|\sigma|=N \in 2 \mathbb{N}+1$, we observe that

$$
E_{-}\left(\epsilon_{i}, z\right) E_{-}\left(-\epsilon_{j}, a z\right) \in \operatorname{End}\left(V_{0}\right)[[z]]
$$


for $1 \leq i, j \leq \nu$ and $a \in \sigma(G)$. From the definition of vertex operators and the fact that $e_{i i}(m, 0) \notin L, m \in 2 \mathbb{Z}+1 \cap N \mathbb{Z}$, we have obtained that the subspace $V_{0}$ is invariant under the action of $L$. As the usual reason, one can easily see that $V_{0}$ is irreducible as $L$-module.

Finally, we will prove that $V_{\nu}(\gamma) \otimes S\left(\mathcal{H}^{-}\right)$is completely reducible as $\widehat{\mathfrak{e u}}_{\nu}\left(\mathcal{R}_{\sigma},{ }^{-}\right)$-module if $|\sigma| \in 2 \mathbb{N}+1$. Let $d$ be the degree derivation on $\mathcal{R}_{\sigma}$ such that $d . t^{m} e^{\alpha}=m t^{m} e^{\alpha}$ for $m \in \mathbb{Z}, \alpha \in G$. Let $\tilde{L}$ be the semi-direct product of the Lie algebra $L$ and the derivation $d$. Extend $\tau$ to a conjugate anti-involution of $\tilde{L}$ by setting $\tau(d)=d$. Let $d_{0}$ be the degree operator on $S\left(\mathcal{H}^{-}\right)$determined by $d_{0} . \epsilon_{i}(m)=m \epsilon_{i}(m)$ for $1 \leq i \leq \nu, m \in-(2 \mathbb{N}+1)$. Then, by letting the action of $d$ as $1 \otimes d_{0}, V$ can be extended to an $\tilde{L}$-module and is unitary with respect to $\tau$. One has a natural $\mathbb{Z}$-graded structure on $\tilde{L}$ and $V$ :

$$
\tilde{L}=\oplus_{n \in \mathbb{Z}} \tilde{L}_{n}, V=\oplus_{m \in-\mathbb{N}} V(m),
$$

with respect to the action of $d$. For any submodule $V^{\prime}$ of $V$, write $V^{\prime}(m)=V(m) \cap V^{\prime}$ for $m \in-\mathbb{N}$ so that $V^{\prime}=\oplus_{m} V^{\prime}(m)$. Note that each graded subspace $V(m)$ is finite dimensional. Therefore, each $\tilde{L}$-submodule of $V$ has a orthogonal compliment being unitary (in this case, each $a \in \sigma(G)$ is a root of unity so that $V$ is unitary by Proposition 4.2).

Let $W$ be the sum of all irreducible $\tilde{L}$-submodules (which contain $V_{0}$ ) and $W^{\prime}$ be its orthogonal compliment. Let $W^{\prime}=\oplus_{m \leq n^{\prime}} W^{\prime}(m)$ with $W^{\prime}\left(n^{\prime}\right) \neq 0$ for some $n^{\prime} \in-\mathbb{N}$. Let $U$ be the $\tilde{L}_{0}$-module generated by $W^{\prime}\left(n^{\prime}\right)$, then $U \subseteq V\left(n^{\prime}\right)$ and is finite dimensional. Notice that $\tau\left(\tilde{L}_{0}\right)=\tilde{L}_{0}$, then $U$ is a finite dimensional unitary $\tilde{L}_{0}$-module and hence completely reducible. Let $U_{0}$ be an irreducible component of $\tilde{L}_{0}$-module $U$ and consider the $\tilde{L}$-module $U^{\prime}$ generated by $U_{0}$. Now, $U^{\prime}$ is an irreducible $\tilde{L}$-submodule of $W^{\prime}$ as $U^{\prime}\left(n^{\prime}\right)=U_{0}$ can't split. But $W$ is supposed to contain all the irreducible $\tilde{L}$-submodules of $V$, a contradiction. Therefore, $V=W$ and $V$ is completely reducible as $\tilde{L}$-module (also as $L$-module).

In the rest of this paper, we will pay our attention to the trivial case that $G=\{1\}$. In this case, the elementary unitary Lie algebra $\widehat{\mathfrak{e}}_{\nu}\left(\mathcal{R}_{\sigma},{ }^{-}\right)$is isomorphic to the twisted affine Kac-Moody algebra of type $A_{\nu-1}^{(2)}$. Let $\Lambda_{j}, 0 \leq j \leq d, d=\left[\frac{\nu}{2}\right]$ be the fundamental integral weight of $A_{\nu-1}^{(2)}$. Following from Theorem 4.4 and the techniques developed by Wakimoto (See [W] Theorem 4.2), we can find that

Corollary 4.5 $M$ is a completely reducible representation for the affine Kac-Moody algebra of type $A_{\nu-1}^{(2)}$ with the action given in Theorem 3.2. If $\nu=2 d$, then $V_{2 d}(\gamma) \otimes S\left(\mathcal{H}_{0}^{-}\right), \gamma \in \mathbb{Z}_{2}^{d+1}$ are the irreducible $\widehat{\mathfrak{e u}}_{\nu}\left(\mathcal{R}_{\sigma},{ }^{-}\right)$-modules with highest weight $\Lambda_{d-1}$ if $\gamma_{1} \cdots \gamma_{d+1}=-1$ or $\Lambda_{d}$ if $\gamma_{1} \cdots \gamma_{d+1}=1$. If $\nu=2 d+1$, then $V_{2 d+1}(\gamma) \otimes S\left(\mathcal{H}_{0}^{-}\right), \gamma \in \mathbb{Z}_{2}^{d+1}$ are the irreducible $\widehat{\mathfrak{e u}}_{\nu}\left(\mathcal{R}_{\sigma}{ }^{-}{ }^{-}\right)$ modules with highest weight $\Lambda_{d}$.

Remark 4.6 The vertex operator representations $\mathbb{C}[\bar{Q}] \otimes S\left(\mathcal{H}_{0}^{-}\right)$for affine Kac-Moody algebra of type $A_{\nu-1}^{(2)}$ have been studied in [W], where the author used a different two cocycle $\varepsilon():, Q \rightarrow Q$ from our's. Explicitly, let $\Pi=\left\{\alpha_{i}=\epsilon_{i}-\epsilon_{i+1}, 1 \leq i \leq \nu-1\right\}$ be the simple root 
system of type $A_{\nu-1}$, then the two-cocycle $\varepsilon($,$) is determined by \varepsilon\left(\alpha_{i}, \alpha_{j}\right)(1 \leq i, j \leq \nu-1)$ and its bi-multiplicative property. The Dynkin diagram of $\Pi$ with orientation corresponds to $\varepsilon$ as follows:

$$
\varepsilon\left(\alpha_{i}, \alpha_{j}\right)= \begin{cases}1, & \text { if } \stackrel{\alpha_{i}}{\bigcirc} \longrightarrow \stackrel{\alpha_{j}}{\bigcirc} \text { or } \alpha_{i} \text { is not connected with } \alpha_{j}, \\ -1, & \text { if } i=j \text { or } \stackrel{\alpha_{i}}{\bigcirc} \longleftarrow \alpha_{j} .\end{cases}
$$

The orientation of Dynkin diagram used in [W] was

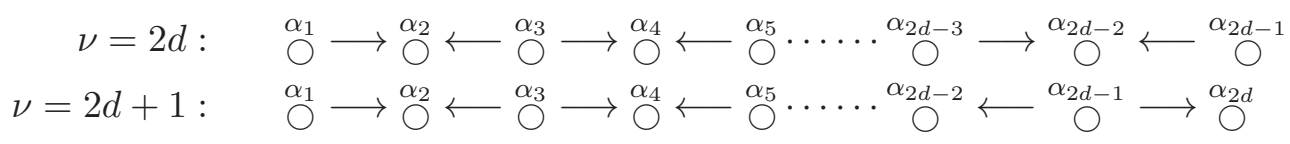

while the orientation used in this paper as follows

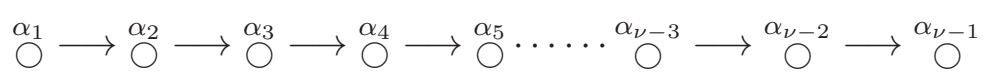

Remark 4.7 Let $G=\mathbb{Z}$ and consider the subalgebra $\mathcal{G}$ of $\widehat{\mathfrak{e}}_{\nu}\left(\mathcal{R}_{\sigma}{ }^{-}{ }^{-}\right)$generated by the elements $e_{i j}(0, m), 1 \leq i \neq j \leq \nu, m \in \mathbb{Z}$. Then, $\mathcal{G}$ is a fixed point subalgebra of affine Kac-Moody algebra $A_{\nu-1}^{(1)}$. But, $\mathcal{G}$ is not isomorphic to the (untwisted or twisted) affine KacMoody algebra. The representation theory of $\mathcal{G}$ are known little so far. It is interesting to see that $\tau(x)=-x$ for all $x \in \mathcal{G}$ if $|\sigma(1)|=1$.

\section{References}

[ABG] B. N. Allison, G. Benkart, Y. Gao, Lie algebras graded by the root system $B C_{r}, r \geq$ 2, Memoirs of the Amer. Math. Soc., 751, Providence R.I., 2002.

[AF] B. N. Allison, J. R. Faulkner, Nonassociative coefficient algebras for Steinberg unitary Lie algebras, J. Algebra, 161 (1993), 1-19.

[B] Y. Billig, Principal vertex operator representations for toroidal Lie algebras, J. Math. Phys., 39 (1998), 3844-3864.

[BGT] S. Berman, Y. Gao, S. Tan, A unified view on vertex operator construction, Israel Journal of Math. 134 (2003), 29-60.

[BJT] S. Berman, E. Jurisich, S. Tan, Beyond Borcherds Lie algebras and inside, Transactions of Amer. Math. Soc., 353 (2001), 1183-1219.

[BS] S. Berman and J. Szmigielski, Principal realization for the extended affine Lie algebra of type $s l_{2}$ with coordinates in a simple quantum torus with two generators, Contemp. Math. 248 (1999), 39-67.

[EM] Rao S. Eswara and R. V. Moody, Vertex representations for N-toroidal Lie algebras and a generalization of the Virasoro algebra, Comm. Math. Phys. 159 (1994), 239264 . 
[FJ] I. Frenkel, N. Jing, Vertex represetations of quatumn affine Lie algebras. Proc. Natl. Acad. Sci., USA 85 (1988), 9373-9377.

[FJW] I. Frenkel, N. Jing and W. Wang, Vertex representations via finite groups and the McKay correspondence. Internat. Math. Res. Notices, 4 (2000), 195-222.

[FK] I. Frenkel, V. Kac, Basic Representations of affine Lie algebras and dual resonance models, Invent. Math., 62 (1980), 23-66.

[FLM] I. B. Frenkel, J. Lepowsky, A. Meurman, Vertex operator algebras and the Monster, Academic Press, Boston, 1988.

[G1] Y. Gao, Steinberg unitary Lie algebras and skew-dihedral homology, J. Algebra, 179 (1996), 261-304.

[G2] Y. Gao, Involutive Lie algebras graded by finite root systems and compact forms of IM algebras, Math. Zeit., 223 (1996) 651-672.

[G3] Y. Gao, Vertex operators arising from the homogeneous realization for $\hat{g l}{ }_{N}$, Communications in Math. Phys., 211 (2000), 23-66.

[G4] Y. Gao, Representations of extented affine Lie algebras coordinatized by certain quantum tori, Compositio Math., 123 (2000), 1-25.

[J] N. Jing, Twisted vertex representations of the quantum affine algebras, Invent. Math., 102 (1990), 663-690.

[KKLW] V. G. Kac, D. A. Kazhdan, J. Lepowsky, and R. L. Wilson, Realization of the basic representations of the Euclidean Lie algebras. Advances in Math., 42 (1981), $83-112$.

[KL] C. Kassel, J.-L. Loday, Extensions centrales d'algèbres de Lie, Ann. Inst. Fourier (Grenoble) 32 (1982), 119-142.

[LW] J. Lepowsky, R. L. Wilson, Construction of the affine Lie algebra $A_{1}^{(1)}$, Commun. Math. Phys., 62 (1978), 43-53.

[MRY] R. V. Moody, S. Eswara Rao, T. Yokomuma, Toroidal lie algebras and vertex representations, Geometriae Dedicata, 35 (1990), 283-307

[S] G. Segal, Unitary representations of some infinite-dimensional groups. Commun. Math. Phys., 80 (1981), 301-342.

[T1] S. Tan, TKK algebras and vertex operator representations, Journal of Algebra, 211 (1999), 298-342.

[T2] S. Tan, Principal construction of the toroidal Lie algebra of type $A_{1}$, Math. Zeit. 230 (1999), 621-657. 
[T3] S. Tan, Vertex operator representations for toroidal Lie algebras of type $B_{l}$, Commun. Algebra, 27 (1999), 3598-3618.

[W] M. Wakimoto, Twisted vertex operators and A-D-E representations, arXiv:math/0111018v2.

[Y] H. Yamada, Extended Affine Lie Algebras and their Vertex Representations, Publ. RIMS, Kyoto University, 25 (1989) 587-603. 